Noname manuscript No.

(will be inserted by the editor)

\title{
Single-crossover recombination in discrete time
}

\author{
Ute von Wangenheim • Ellen Baake · Michael Baake
}

the date of receipt and acceptance should be inserted later

\begin{abstract}
Modelling the process of recombination leads to a large coupled nonlinear dynamical system. Here, we consider a particular case of recombination in discrete time, allowing only for single crossovers. While the analogous dynamics in continuous time admits a closed solution [3], this no longer works for discrete time. A more general model (i.e. without the restriction to single crossovers) has been studied before [5, 9, 10] and was solved algorithmically by means of Haldane linearisation. Using the special formalism introduced in [3], we obtain further insight into the single-crossover dynamics and the particular difficulties that arise in discrete time. We then transform the equations to a solvable system in a two-step procedure: linearisation followed by diagonalisation. Still, the coefficients of the second step must be determined in a recursive manner, but once this is done for a given system, they allow for an explicit solution valid for all times.
\end{abstract}

Keywords population genetics · recombination dynamics · Möbius linearisation · diagonalisation · linkage disequilibria

Mathematics Subject Classification (2000) 92D10 $\cdot 37$ N30 $\cdot$ 06A07 $\cdot 60 J 05$

\section{Introduction}

The dynamics of the genetic composition of populations evolving under recombination has been a long-standing subject of research. The traditional models assume random mating, non-overlapping generations (meaning discrete time), and populations so large that stochastic fluctuations may be neglected and a law of large numbers (or infinite-population limit) applies. Even this highly idealised setting leads to models that are notoriously difficult to treat and solve, namely, to large systems of coupled, nonlinear difference equations. Here, the nonlinearity is due to the random mating of the partner individuals involved in sexual reproduction.

Ute von Wangenheim

Technische Fakultät, Universität Bielefeld, Box 100131, 33501 Bielefeld, Germany

E-mail: uvonwang@techfak.uni-bielefeld.de

Ellen Baake

Technische Fakultät, Universität Bielefeld, Box 100131, 33501 Bielefeld, Germany

E-mail: ebaake@techfak.uni-bielefeld.de

Michael Baake

Fakultät für Mathematik, Universität Bielefeld, Box 100131, 33501 Bielefeld, Germany

E-mail: mbaake@math.uni-bielefeld.de 
Elucidating the underlying structure and finding solutions to these equations has been a challenge to theoretical population geneticists for nearly a century now. The first studies go back to Jennings [13] in 1917 and Robbins [17] in 1918. Building on [13], Robbins solved the dynamics for two diallelic loci (to be called sites from now on) and gave an explicit formula for the (haplo)type frequencies as functions of time. Geiringer [11] investigated the general recombination model for an arbitrary number of loci and for arbitrary 'recombination distributions' (meaning collections of probabilities for the various partitionings of the sites that may occur during recombination) in 1944 . She was the first to state the general form of the solution of the recombination equation (as a convex combination of all possible products of certain marginal frequencies derived from the initial population) and developed a method for the recursive evaluation of the corresponding coefficients. This simplifies the calculation of the type frequencies at any time compared to the direct evaluation through successive iteration of the dynamical system. Even though she worked out the method for the general case in principle, its evaluation becomes quite involved for more than three sites.

Her work was followed by Bennett [5] in 1954. He introduced a multilinear transformation of the type frequencies to certain functions that he named principal components. They correspond to linear combinations of certain correlation functions that transform the dynamical system (exactly) into a linear one. The new variables decay independently and geometrically for all times, whence they decouple and diagonalise the dynamics. They therefore provide an elegant solution in principle, but the price to be paid is that the coefficients of the transformation must be constructed via recursions that involve the parameters of the recombination model. Bennett worked this method out for up to six sites, but did not give an explicit method for an arbitrary number of sites. The approach was later completed within the systematic framework of genetic algebras, where it became known as Haldane linearisation, compare [14, 15]. But, in fact, Bennett's program may be completed outside this abstract framework, as was shown by Dawson [9, 10], who derived a general and explicit recursion for the coefficients of the principal components. However, the proofs are somewhat technical and do not reveal the underlying mathematical structure. It is the aim of this paper to provide a more systematic, but still elementary, approach that exploits the inherent (multi)linear and combinatorial structure of the problem - at least for one particular, but biologically relevant, special case, which will now be described. Our special case is obtained by the restriction to single crossovers, which leads to what we call single-crossover recombination (SCR). This is the extreme case of the biological phenomenon of interference, and describes the situation where a crossover event completely inhibits any other crossover event in the same generation, at least within the genomic region considered. Surprisingly, the corresponding dynamics in continuous time can be solved in closed form [2, 3]. Again, a crucial ingredient is a transformation to certain correlation functions (or linkage disequilibria) that linearise and diagonalise the system. Luckily, in this case, the corresponding coefficients are independent of the recombination parameters, and the transformation is available explicitly.

Motivated by this result, we now investigate the analogous single-crossover dynamics in discrete time. The paper is organised as follows. We first describe the discrete-time model and the general framework (Section 2) and then recapitulate the essentials of the continuous-time model and its solution (Section 3). Section 4 returns to discrete time. We first analyse explicitly the cases of two, three, and four sites. For two and three sites, the dynamics is analogous to that in continuous time (and, in particular, available in closed form), but differs from then on. This is because a certain linearity present in continuous time is now lost. The transformations used in continuous time are therefore not sufficient to both linearise and diagonalise the discrete-time dynamics. They do, however, lead to a linearisation; this is worked out in Sections 5 and 6. The resulting linear system has a triangular structure that can be diagonalised in a second step in a recursive way (Section 7). We summarise and discuss our results in Section 8 . An explicit example is worked out in the Appendix. 


\section{Preliminaries and notation}

Let us briefly recall the recombination model described in [3] and the special notation introduced there, as the remainder of this paper critically depends on it. A chromosome (of length $n+1$, say) is represented as a linear arrangement of the $n+1$ sites of the set $S=\{0,1, \ldots, n\}$. Sites are discrete positions on a chromosome that may be interpreted as gene or nucleotide positions. A set $X_{i}$ collects the possible elements (such as alleles or nucleotides) at site $i$. For convenience, we restrict ourselves to finite sets $X_{i}$ in this paper, though much of the theory can be extended to the case that each $X_{i}$ is a locally compact space, which can be of importance for applications in quantitative genetics. A type is now defined as a sequence $\left(x_{0}, x_{1}, \ldots, x_{n}\right) \in X_{0} \times X_{1} \times \cdots \times X_{n}=: X$, where $X$ denotes the (finite) type space.

Recombination events take place at the so-called links between neighbouring sites, collected into the set $L=\left\{\frac{1}{2}, \frac{3}{2}, \ldots, \frac{2 n-1}{2}\right\}$, where link $\alpha=\frac{2 i+1}{2}$ is the link between sites $i$ and $i+1$. Since we only consider single crossovers here, each individual event yields an exchange of the sites either before or after the respective link between the two types involved. A recombination event at link $\frac{2 i+1}{2}$ that involves $x=\left(x_{0}, \ldots, x_{n}\right)$ and $y=\left(y_{0}, \ldots, y_{n}\right)$ thus results in the types $\left(x_{0}, \ldots, x_{i}, y_{i+1}, \ldots, y_{n}\right)$ and $\left(y_{0}, \ldots, y_{i}, x_{i+1}, \ldots, x_{n}\right)$, with both pairs considered as unordered.

Although one is ultimately interested in the stochastic process defined by recombination acting on populations of finite size, compare [4] and references therein, we restrict ourselves to the deterministic limit of infinite population size here, also known as infinite population limit (IPL). Consequently, we are not looking at the individual dynamics, but at the induced dynamics on the probability distribution on the type space $X$. Let $\mathcal{P}(X)$ denote the convex space of all possible probability distributions on $X$. As $X$ is finite, a probability distribution can be written as a vector $p=(p(x))_{x \in X}$, where $p(x)$ denotes the relative frequency of type $x$ in the population.

Let us look at the time evolution of the relative frequencies $p_{t}(x)$ of types $x=\left(x_{0}, \ldots, x_{n}\right)$ when starting from a known initial distribution $p_{0}$ of the population at time $t=0$. In discrete time, it is given by the following collection of recombination equations for all $x \in X$ :

$$
\begin{aligned}
p_{t+1}(x)= & \sum_{\alpha \in L} \rho_{\alpha} p_{t}\left(x_{0}, x_{1}, \ldots, x_{\lfloor\alpha\rfloor}, *, *, \ldots, *\right) p_{t}\left(*, *, \ldots, *, x_{\lceil\alpha\rceil}, x_{\lceil\alpha\rceil+1}, \ldots, x_{n}\right) \\
& +\left(1-\sum_{\alpha \in L} \rho_{\alpha}\right) p_{t}(x), \quad \text { with } t \in \mathbb{N}_{0},
\end{aligned}
$$

where the coefficients $\rho_{\alpha}, \alpha \in L$, are the probabilities for a crossover at link $\alpha$. Consequently, we must have $\rho_{\alpha} \geq 0$ and $\sum_{\alpha \in L} \rho_{\alpha} \leq 1$, where $\rho_{\alpha}>0$ is assumed from now on without loss of generality (when $\rho_{\alpha}=0$, the set $X_{\alpha-\frac{1}{2}} \times X_{\alpha+\frac{1}{2}}$ can be considered as a space for an effective site that comprises $i=\alpha-\frac{1}{2}$ and $\left.i=\alpha+\frac{1}{2}\right)$. When the $\rho_{\alpha}$ do not sum to 1 , the remainder is the probability that no crossover occurs, which is taken care of by the last term in the equation. Moreover, $\lfloor\alpha\rfloor(\lceil\alpha\rceil)$ denotes the largest integer below (the smallest above) $\alpha$ and the star $*$ at site $i$ stands for $X_{i}$, and thus indicates marginalisation over site $i$.

An important step to solve the large nonlinear coupled system of equations (1) lies in its reformulation in a more compact way with the help of certain recombination operators. To construct them, we need the canonical projection operator $\pi_{i}: X \longrightarrow X_{i}$, defined by $x \mapsto \pi_{i}(x)=x_{i}$ as usual. Likewise, for any index set $J \subseteq S$, the projector $\pi_{J}$ is defined as $\pi_{J}: X \longrightarrow X_{J}:=\chi_{i \in J} X_{i}$. We will frequently use

$$
\pi_{<\alpha}:=\pi_{\{0, \ldots,\lfloor\alpha\rfloor\}} \quad \text { and } \quad \pi_{>\alpha}:=\pi_{\{\lceil\alpha\rceil, \ldots, n\}} .
$$

These can be understood as cut-and-forget operators since they 'cut out' the leading and the trailing segment of a type $x$, respectively, and 'forget' about the rest. The projectors induce linear mappings from $\mathcal{P}(X)$ to $\mathcal{P}\left(X_{J}\right)$ by $p \mapsto \pi_{J} . p:=p \circ \pi_{J}^{-1}$, where $\pi_{J}^{-1}$ denotes the preimage under $\pi_{J}$ and $\circ$ 
indicates composition of mappings. The operation . (not to be confused with a multiplication sign) is known as the pullback of $\pi_{J}$ with respect to $p$. Consequently, $\pi_{J} . p$ is simply the marginal distribution of $p$ with respect to the sites of $J$.

Now consider recombination at link $\alpha$, performed on the entire population. Since the resulting population consists of randomly chosen leading segments relinked with randomly chosen trailing segments, it may be described through the (elementary) recombination operator (or recombinator for short) $R_{\alpha}: \mathcal{P}(X) \longrightarrow \mathcal{P}(X)$, defined by $p \mapsto R_{\alpha}(p)$ with

$$
R_{\alpha}(p):=\left(\pi_{<\alpha} \cdot p\right) \otimes\left(\pi_{>\alpha} \cdot p\right),
$$

where $\otimes$ denotes the product measure and reflects the independent combination of both marginals $\pi_{<\alpha} . p$ and $\pi_{>\alpha} . p$. Note that the recombinators are structural operators that do not depend on the recombination probabilities.

Before we rewrite the recombination equations in terms of these recombinators, let us recall some of their elementary properties, see [3] for proofs. First of all, the elementary recombinators $R_{\alpha}$ are idempotents and commute with one another on $\mathcal{P}(X)$. This permits the consistent definition of composite recombinators

$$
R_{G}:=\prod_{\alpha \in G} R_{\alpha}
$$

for arbitrary subsets $G \subseteq L$. In particular, one has $R_{\varnothing}=\mathbb{1}$ and $R_{\{\alpha\}}=R_{\alpha}$.

Proposition 1 On $\mathcal{P}(X)$, the elementary recombinators are commuting idempotents. For $\alpha \leq \beta$, they satisfy

$$
\pi_{<\alpha \cdot}\left(R_{\beta}(p)\right)=\pi_{<\alpha \cdot p} \quad \text { and } \quad \pi_{>\alpha \cdot}\left(R_{\beta}(p)\right)=\left(\pi_{\{\lceil\alpha\rceil, \ldots,\lfloor\beta\rfloor\}} \cdot p\right) \otimes\left(\pi_{>\beta} \cdot p\right) ;
$$

likewise, for $\alpha \geq \beta$,

$$
\pi_{>\alpha \cdot}\left(R_{\beta}(p)\right)=\pi_{>\alpha \cdot} \cdot p \quad \text { and } \quad \pi_{<\alpha \cdot}\left(R_{\beta}(p)\right)=\left(\pi_{<\beta} \cdot p\right) \otimes\left(\pi_{\{\lceil\beta\rceil, \ldots,\lfloor\alpha\rfloor\}} \cdot p\right) .
$$

Furthermore, the composite recombinators satisfy

$$
R_{G} R_{H}=R_{G \cup H}
$$

for arbitrary $G, H \subseteq L$.

These properties can be understood intuitively as well: (4) says that recombination at or after link $\alpha$ does not affect the marginal frequencies at sites before $\alpha$, whereas the marginal frequencies at the sites after $\alpha$ change into the product measure (and vice versa in (5)). Furthermore, repeated recombination at link $\alpha$ does not change the situation any further (recombinators are idempotents) and the formation of the product measure with respect to $\geq 2$ links does not depend on the order in which the links are affected. As we shall see below, these properties of the recombinators are crucial for finding a solution of the SCR dynamics, both in continuous and in discrete time.

\section{$3 \mathrm{SCR}$ in continuous time}

Let us briefly review the SCR dynamics in continuous time, as its structure will be needed below. Making use of the recombinators introduced above, the dynamics (in the IPL) is described by a system of differential equations for the time evolution of the probability distribution (or measure), starting from an initial condition $p_{0}$ at $t=0$. It reads [3]

$$
\dot{p}_{t}=\sum_{\alpha \in L} \widetilde{\rho}_{\alpha}\left(R_{\alpha}-\mathbb{1}\right)\left(p_{t}\right),
$$

where $\widetilde{\rho}_{\alpha}$ is now the rate for a crossover at link $\alpha$. Though (7) describes a coupled system of nonlinear differential equations, the closed solution for its Cauchy (or initial value) problem is available [2, 3]: 
Theorem 1 The solution of the recombination equation (7) with initial value $p_{0}$ can be given in closed form as

$$
p_{t}=\sum_{G \subseteq L} \tilde{a}_{G}(t) R_{G}\left(p_{0}\right)
$$

with the coefficient functions

$$
\widetilde{a}_{G}(t)=\prod_{\alpha \in L \backslash G} \exp \left(-\widetilde{\rho}_{\alpha} t\right) \prod_{\beta \in G}\left(1-\exp \left(-\widetilde{\rho}_{\beta} t\right)\right)
$$

These are non-negative functions, which satisfy $\sum_{G \subseteq L} \widetilde{a}_{G}(t)=1$ for all $t \geq 0$.

The coefficient functions can be interpreted probabilistically. Given an individual sequence in the population, $\widetilde{a}_{G}(t)$ is the probability that the set of links that have seen at least one crossover event until time $t$ is precisely the set $G$. Note that the product structure of the $\widetilde{a}_{G}(t)$ implies independence of links, a decisive feature of the single-crossover dynamics in continuous time, as we shall see later on. By (8), $p_{t}$ is always a convex combination of the probability measures $R_{G}\left(p_{0}\right)$ with $G \subseteq L$. Consequently, given an initial condition $p_{0}$, the entire dynamics takes place on the closed simplex (within $\mathcal{P}(X)$ ) that is given by $\operatorname{conv}\left\{R_{G}\left(p_{0}\right) \mid G \subseteq L\right\}$, where $\operatorname{conv}(A)$ denotes the convex hull of $A$.

It is surprising that a closed solution for the dynamics (7) can be given explicitly, and this suggests the existence of an underlying linear structure [2], which is indeed the case and well known from similar equations, compare [14]. In the context of the formulation with recombinators, it can be stated as follows, compare [3] for details.

Theorem 2 Let $\left\{c_{G^{\prime}}^{\left(L^{\prime}\right)}(t) \mid \varnothing \subseteq G^{\prime} \subseteq L^{\prime} \subseteq L\right\}$ be a family of non-negative functions with $c_{G}^{(L)}(t)=$ $c_{G_{1}}^{\left(L_{1}\right)}(t) c_{G_{2}}^{\left(L_{2}\right)}(t)$, valid for any partition $L=L_{1} \dot{\cup} L_{2}$ of the set $L$ and all $t \geq 0$, where $G_{i}:=G \cap L_{i}$. Assume further that these functions satisfy $\sum_{H \subseteq L^{\prime}} c_{H}^{\left(L^{\prime}\right)}(t)=1$ for any $L^{\prime} \subseteq L$ and $t \geq 0$. If $v \in \mathcal{P}(X)$ and $H \subseteq L$, one has the identity

$$
R_{H}\left(\sum_{G \subseteq L} c_{G}^{(L)}(t) R_{G}(v)\right)=\sum_{G \subseteq L} c_{G}^{(L)}(t) R_{G \cup H}(v),
$$

which is then satisfied for all $t \geq 0$.

Here, the upper index specifies the respective set of links. So far, Theorem 2 depends crucially on the product structure of the functions $c_{G}^{(L)}(t)$, but we will show later how this assumption can be relaxed. In any case, the coefficient functions $\widetilde{a}_{G}(t)$ of (9) satisfy the conditions of Theorem 2 . The result then means that the recombinators act linearly along solutions (8) of the recombination equation (7). Denoting $\varphi_{t}$ as the flow of Eq. (7), Theorem 2 thus has the following consequence.

Corollary 1 On $\mathcal{P}(X)$, the forward flow of (7) commutes with all recombinators, which means that $R_{G} \circ \varphi_{t}=\varphi_{t} \circ R_{G}$ holds for all $t \geq 0$ and all $G \subseteq L$.

The conventional approach to solve the recombination dynamics consists in transforming the type frequencies to certain functions which diagonalise the dynamics, see [5, 9, 10, 14] and references therein for more. From now on, we will call these functions principal components after Bennett [5]. For the single-crossover dynamics in continuous time, they have a particularly simple structure: they are given by certain correlation functions, or linkage disequilibria (LDE), which play an important role in biological applications. They have a counterpart at the level of operators on $\mathcal{P}(X)$.

Namely, let us define $L D E$ operators on $\mathcal{P}(X)$ as linear combinations of recombinators via

$$
T_{G}:=\sum_{H \supseteq G}(-1)^{|H-G|} R_{H}, \quad \text { with } G \subseteq L,
$$


so that the inverse relation is given by

$$
R_{H}=\sum_{G \supseteq H} T_{G}
$$

due to the combinatorial Möbius inversion formula, compare [1]. Let us note for further use that, by Eq. (6) in Proposition 1 $T_{G} \circ R_{G}=T_{G}$. Note also that, for a probability measure $p$ on $X, T_{G}(p)$ is a signed measure on $X$; in particular, it need not be positive. The LDEs are given by certain components of the $T_{G}(p)$ - see [3, 4] for more. In the continuous-time single-crossover setting, it was shown in [3] that, if $p_{t}$ is the solution (8), the $T_{G}\left(p_{t}\right)$ satisfy

$$
\frac{\mathrm{d}}{\mathrm{d} t} T_{G}\left(p_{t}\right)=-\left(\sum_{\alpha \in L \backslash G} \widetilde{\rho}_{\alpha}\right) T_{G}\left(p_{t}\right), \quad \text { for all } G \subseteq L,
$$

which is a decoupled system of homogeneous linear differential equations, with the standard exponential solution. That is, the LDE operators both linearise and diagonalise the system, and the LDEs are thus, at the same time, principal components.

A straightforward calculation now reveals that the solution (8) can be rewritten as

$$
p_{t}=\sum_{G \subseteq L} \widetilde{a}_{G}(t) R_{G}\left(p_{0}\right)=\sum_{K \subseteq L} b_{K}(t) T_{K}\left(p_{0}\right)=\sum_{K \subseteq L} T_{K}\left(p_{t}\right),
$$

where the new coefficient functions are given by

$$
b_{K}(t):=\exp \left(-\sum_{\alpha \in L \backslash K} \tilde{\rho}_{\alpha} t\right)
$$

At this point, it is important to notice the rather simple structure of the LDE operators, which do not depend on the crossover rates. Moreover, the transformation between recombinators and LDE operators is directly given by the Möbius formula, see Eqs. (10) and (11). This is a significant simplification in comparison with previous results, compare [5, 9, 10, 11], where the coefficients of the transformation generally depend on the crossover rates and must be determined recursively.

Below, we shall see that the SCR dynamics in continuous time is indeed a special case, and that the above results cannot be transferred directly to the corresponding dynamics in discrete time. Nevertheless, part of the continuous-time structure prevails and offers a useful entry point for the solution of the discrete-time counterpart.

\section{$4 \mathrm{SCR}$ in discrete time}

Employing recombinators, the SCR equations (1) in discrete time with a given initial distribution $p_{0}$ can be compactly rewritten as

$$
p_{t+1}=p_{t}+\sum_{\alpha \in L} \rho_{\alpha}\left(R_{\alpha}-\mathbb{1}\right)\left(p_{t}\right)=: \Phi\left(p_{t}\right)
$$

As indicated, the nonlinear operator of the right-hand side of (14) is denoted by $\Phi$ from now on. We aim at a closed solution of (14), namely for $p_{t}=\Phi^{t}\left(p_{0}\right)$ with $t \in \mathbb{N}_{0}$. Based on the result for the continuous-time model, the solution is expected to be of the form

$$
p_{t}=\Phi^{t}\left(p_{0}\right)=\sum_{G \subseteq L} a_{G}(t) R_{G}\left(p_{0}\right),
$$

with non-negative $a_{G}(t), G \subseteq L, \sum_{G \subseteq L} a_{G}(t)=1$, describing the (unknown) coefficient functions arising from the dynamics. This representation of the solution was first stated by Geiringer [11]. In particular, also the discrete-time dynamics takes place on the $\operatorname{simplex} \operatorname{conv}\left\{R_{G}\left(p_{0}\right) \mid G \subseteq L\right\}$. 
We are particularly interested in whether a discrete-time equivalent to Corollary 1 exists, that is, whether all recombinators $R_{G}$ commute with $\Phi$. This is of importance since it would allow for a diagonalisation of the dynamics via the LDE operators (10). To see this, assume for a moment that $R_{\alpha} \circ \Phi=\Phi \circ R_{\alpha}$ for all $\alpha \in L$, and thus $R_{G} \circ \Phi=\Phi \circ R_{G}$ for all $G \subseteq L$. Noting that, when $\alpha \in G \subseteq H$, Eq. (6) from Proposition[1 implies that $\left(R_{\alpha}-\mathbb{1}\right) R_{H}=R_{H \cup\{\alpha\}}-R_{H}=R_{H}-R_{H}=0$, we see that the assumption above would lead to

$$
\begin{aligned}
& T_{G} \circ \Phi=\sum_{H \supseteq G}(-1)^{|H-G|} R_{H} \circ \Phi=\sum_{H \supseteq G}(-1)^{|H-G|} \Phi \circ R_{H} \\
& =\sum_{H \supseteq G}(-1)^{|H-G|} R_{H}+\sum_{H \supseteq G}(-1)^{|H-G|} \sum_{\alpha \in L} \rho_{\alpha}\left(R_{\alpha}-\mathbb{1}\right) R_{H} \\
& =T_{G}+\sum_{H \supseteq G}(-1)^{|H-G|} \sum_{\alpha \in L \backslash G} \rho_{\alpha}\left(R_{\alpha}-\mathbb{1}\right) R_{H} \\
& =\left(1-\sum_{\alpha \in L \backslash G} \rho_{\alpha}\right) T_{G}+\sum_{\alpha \in L \backslash G} \rho_{\alpha} \sum_{H \supseteq G}\left((-1)^{|H-G|} R_{H \cup\{\alpha\}}+(-1)^{|H \cup\{\alpha\}-G|} R_{H \cup\{\alpha\}}\right) \\
& =\left(1-\sum_{\alpha \in L \backslash G} \rho_{\alpha}\right) T_{G},
\end{aligned}
$$

so that, indeed, all $T_{G}\left(p_{t}\right)$ would decay geometrically. This wishful calculation is badly smashed by the nonlinear nature of the recombinators, and the remainder of this paper is concerned with true identities that repair the damage.

To get an intuition for the dynamics in discrete time, let us first take a closer look at the discretetime model with two, three, and four sites.

\subsection{Two and three sites}

For two sites, one simply has $S=\{0,1\}$ and $L=\left\{\frac{1}{2}\right\}$, so that only one non-trivial recombinator exists, $R=R_{\frac{1}{2}}$, with corresponding recombination probability $\rho=\rho_{\frac{1}{2}}$. Consequently, the SCR equation simplifies to

$$
p_{t+1}=\Phi\left(p_{t}\right)=\rho R\left(p_{t}\right)+(1-\rho) p_{t},
$$

where $p_{t}$ is a $|X|$-dimensional probability vector. The solution is given by

$$
p_{t}=a(t) p_{0}+(1-a(t)) R\left(p_{0}\right)
$$

with $a(t)=a_{\varnothing}(t)=(1-\rho)^{t}$. This formula is easily verified by induction [18]. Thus, in analogy with the SCR dynamics in continuous time, the solution is available in closed form, and the coefficient functions allow an analogous probabilistic interpretation. Furthermore, it is easily seen that the recombinators $R_{\varnothing}=\mathbb{1}$ and $R_{\frac{1}{2}}=R$ commute with $\Phi$ and therefore with $\Phi^{t}$ for all $t \in \mathbb{N}_{0}$. For two sites, the analogue of Corollary 1 thus holds in discrete time. As a consequence, the LDE operators from (10) decouple and linearise the system (16). At the level of the component LDEs, this is common knowledge in theoretical population genetics; compare [12, Chap.3].

Similarly, the recombination equation (11) for three sites can be solved explicitly as well. An elementary calculation (applying the iteration and comparing coefficients) shows that the corresponding coefficient functions $a_{G}(t)$ follow the linear recursion

$$
\left(\begin{array}{c}
a_{\varnothing}(t+1) \\
a_{\frac{1}{2}}(t+1) \\
a_{\frac{3}{2}}(t+1) \\
a_{\left\{\frac{1}{2}, \frac{3}{2}\right\}}(t+1)
\end{array}\right)=\left(\begin{array}{cccc}
1-\rho_{\frac{1}{2}}-\rho_{\frac{3}{2}} & 0 & 0 & 0 \\
\rho_{\frac{1}{2}} & 1-\rho_{\frac{3}{2}} & 0 & 0 \\
\rho_{\frac{3}{2}} & 0 & 1-\rho_{\frac{1}{2}} & 0 \\
0 & \rho_{\frac{3}{2}} & \rho_{\frac{1}{2}} & 1
\end{array}\right)\left(\begin{array}{c}
a_{\varnothing}(t) \\
a_{\frac{1}{2}}(t) \\
a_{\frac{3}{2}}(t) \\
a_{\left\{\frac{1}{2}, \frac{3}{2}\right\}}(t)
\end{array}\right),
$$


with solution

$$
\begin{aligned}
a_{\varnothing}(t) & =\left(1-\rho_{\frac{1}{2}}-\rho_{\frac{3}{2}}\right)^{t}, \\
a_{\frac{1}{2}}(t) & =\left(1-\rho_{\frac{3}{2}}\right)^{t}-\left(1-\rho_{\frac{1}{2}}-\rho_{\frac{3}{2}}\right)^{t}, \\
a_{\frac{3}{2}}(t) & =\left(1-\rho_{\frac{1}{2}}\right)^{t}-\left(1-\rho_{\frac{1}{2}}-\rho_{\frac{3}{2}}\right)^{t}, \\
a_{\left\{\frac{1}{2}, \frac{3}{2}\right\}}(t) & =1-\left(1-\rho_{\frac{3}{2}}\right)^{t}-\left(1-\rho_{\frac{1}{2}}\right)^{t}+\left(1-\rho_{\frac{1}{2}}-\rho_{\frac{3}{2}}\right)^{t} .
\end{aligned}
$$

These coefficient functions have the same probabilistic interpretation as the corresponding $\widetilde{a}_{G}(t)$, $G \subseteq L$, in the continuous-time model, so that $a_{G}(t)$ is the probability that the links that have been involved in recombination until time $t$ are exactly those of the set $G$.

But there is a crucial difference. Recall that, in continuous time, single crossovers imply independence of links, which is expressed in the product structure of the coefficient functions $\widetilde{a}_{G}(t)($ see (9) $)$. This independence is lost in discrete time, where a crossover event at one link forbids any other cut at other links in the same time step. Consequently, already for three sites, the coefficients of the discrete-time dynamics fail to show the product structure used in Theorem 2.

But even though Corollary 1 concerning the forward flow of (17), is a consequence of Theorem 2 which, in turn, is based upon the product structure of the coefficients, a short calculation reveals that $R_{G} \circ \Phi=\Phi \circ R_{G}$ still holds for the discrete-time model with three sites for all $G \subseteq\left\{\frac{1}{2}, \frac{3}{2}\right\}$. As a consequence, just as in the case of two sites, the $T_{G}$ linearise and decouple the dynamics, which is well-known to the experts, see [5, 6] for more.

To summarise: despite the loss of independence of links, an explicit solution of the discrete-time recombination dynamics is still available, and a linearisation and diagonalisation of the dynamics can be achieved with the methods developed for the continuous-time model, that is, a transformation to a solvable system via the $T_{G}$. However, things will become more complex if we go to four sites and beyond. In particular, there is no equivalent to Corollary 1 i.e., in general, the recombinators do not commute with $\Phi$, and we have to search for a new transformation that replaces (10), as will be explained next.

\subsection{Four sites}

The complication with four sites originates from the fact that $R_{\frac{3}{2}} \circ \Phi \neq \Phi \circ R_{\frac{3}{2}}$, so that the property described by Corollary 1 for continuous time is lost here. Consequently, the $T_{G}$ fail the desired properties. In particular, one finds

$$
T_{\varnothing}(\Phi(p))=\left(1-\rho_{\frac{1}{2}}-\rho_{\frac{3}{2}}-\rho_{\frac{5}{2}}\right) T_{\varnothing}(p)-\rho_{\frac{1}{2}} \rho_{\frac{5}{2}} T_{\frac{3}{2}}(p)
$$

so that an explicit solution of the model cannot be obtained as before.

This raises the question why four sites are more difficult than three sites, even though independence of links has already been lost with three sites. To answer this, we look at the time evolution of the coefficient functions $a_{G}(t), G \subseteq L$. For this purpose, let us return to the general model with an arbitrary number of sites.

\subsection{General case}

We now consider an arbitrary (but finite) set $S$ with the corresponding link set $L$. For each $G \subseteq L$, we use the following abbreviations:

$$
\begin{array}{ll}
G_{<\alpha}:=\{i \in G \mid i<\alpha\}, & G_{>\alpha}:=\{i \in G \mid i>\alpha\} \\
L_{\leq \alpha}:=\{i \in L \mid i \leq \alpha\}, & L_{\geq \alpha}:=\{i \in L \mid i \geq \alpha\} .
\end{array}
$$


Furthermore, we set $\eta:=1-\sum_{\alpha \in L} \rho_{\alpha}$. We then obtain

Theorem 3 For all $G \subseteq L$ and $t \in \mathbb{N}_{0}$, the coefficient functions $a_{G}(t)$ evolve according to

$$
a_{G}(t+1)=\eta a_{G}(t)+\sum_{\alpha \in G} \rho_{\alpha}\left(\sum_{H \subseteq L \geq \alpha} a_{G_{<\alpha} \cup H}(t)\right)\left(\sum_{K \subseteq L_{\leq \alpha}} a_{K \cup G_{>\alpha}}(t)\right)
$$

with initial condition $a_{G}(0)=\delta_{G, \varnothing}$.

Proof Geiringer [11] already explained in words how to derive this general recursion, and illustrated it with the four-site example; we give a proof via our operator formalism. Using (15), the recombination equation for $p_{t+1}$ reads

$$
\begin{aligned}
p_{t+1}= & \sum_{G \subseteq L} a_{G}(t+1) R_{G}\left(p_{0}\right)=\Phi\left(p_{t}\right)=\Phi\left(\sum_{G \subseteq L} a_{G}(t) R_{G}\left(p_{0}\right)\right) \\
= & \sum_{\alpha \in L} \rho_{\alpha}\left(\left(\pi_{<\alpha} \cdot\left(\sum_{H \subseteq L} a_{H}(t) R_{H}\left(p_{0}\right)\right)\right) \otimes\left(\pi_{>\alpha} \cdot\left(\sum_{K \subseteq L} a_{K}(t) R_{K}\left(p_{0}\right)\right)\right)\right) \\
& +\eta\left(\sum_{G \subseteq L} a_{G}(t) R_{G}\left(p_{0}\right)\right)
\end{aligned}
$$

where each product term in the first sum can be calculated as

$$
\begin{aligned}
\left(\pi_{<\alpha} \cdot\left(\sum_{H \subseteq L} a_{H}(t) R_{H}\left(p_{0}\right)\right)\right) \otimes\left(\pi_{>\alpha} \cdot\left(\sum_{K \subseteq L} a_{K}(t) R_{K}\left(p_{0}\right)\right)\right) & \\
= & \sum_{H, K \subseteq L} a_{H}(t) a_{K}(t)\left(\left(\pi_{<\alpha} \cdot R_{H}\left(p_{0}\right)\right) \otimes\left(\pi_{>\alpha} \cdot R_{K}\left(p_{0}\right)\right)\right) \\
& =\sum_{H, K \subseteq L} a_{H}(t) a_{K}(t)\left(\left(\pi_{<\alpha} \cdot R_{H_{<\alpha} \cup K_{>\alpha}}\left(p_{0}\right)\right) \otimes\left(\pi_{>\alpha} \cdot R_{H_{<\alpha} \cup K_{>\alpha}}\left(p_{0}\right)\right)\right) \\
& =\sum_{H, K \subseteq L} a_{H}(t) a_{K}(t)\left(R_{\alpha}\left(R_{H_{<\alpha} \cup K_{>\alpha}}\left(p_{0}\right)\right)\right),
\end{aligned}
$$

where we use the linearity of the projectors in the first step, and Eqs. (4) and (5) from Proposition 1 in the second (more precisely, we use the left parts of Eqs. (4) and (5), reading them both forward and backward). Insert this into the expression for $p_{t+1}$ and rearrange the sums for a comparison of coefficients of $R_{G}$ with $G \subseteq L$. Comparison of coefficients is justified by the observation that, for generic $p_{0}$ and generic site spaces, the vectors $R_{G}\left(p_{0}\right)$ with $G \subseteq L$ are the extremal vectors of the closed simplex $\operatorname{conv}\left\{R_{K}\left(p_{0}\right) \mid K \subseteq L\right\}$. They are the vectors that (generically) cannot be expressed as non-trivial convex combination within the simplex, and hence the vertices of the latter (in cases with degeneracies, one reduces the simplex in the obvious way). If $G=\varnothing$, we only have $\eta a_{\varnothing}(t)$ as coefficient for $R_{\varnothing}$. Otherwise, we get additional contributions for each $\alpha \in G$, namely, from those $H, K \subseteq L$ for which $H_{<\alpha}=G_{<\alpha}$ and $K_{>\alpha}=G_{>\alpha}$, while $H_{\geq \alpha}$ and $K_{\leq \alpha}$ can be any subset of $L_{\geq \alpha}$ and $L_{\leq \alpha}$, respectively. Hence, the term belonging to $R_{G}\left(p_{0}\right)$ reads

$$
\sum_{\alpha \in G} \rho_{\alpha}\left(\sum_{H \subseteq L \geq \alpha} \sum_{K \subseteq L_{\leq \alpha}} a_{G_{<\alpha} \cup H}(t) a_{K \cup G_{>\alpha}}(t)\right)+\eta a_{G}(t)
$$

and the assertion follows. 
The $a_{G}(t)$ have the same probabilistic interpretation as the $\widetilde{a}_{G}(t)$ (9) from the continuous-time model, and the above iteration can be understood intuitively as well: A type $x$ resulting from recombination at link $\alpha$ is composed of two segments $x_{<\alpha}$ and $x_{>\alpha}$. These segments themselves may have been pieced together in previous recombination events already, and the iteration explains the possible cuts these segments may carry along. The first term in the product stands for the type delivering the leading segment (which may bring along arbitrary cuts in the trailing segment), the second for the type delivering the trailing one (here any leading segment is allowed). The term $\eta a_{G}(t)$ covers the case of no recombination.

Note that the above iteration is generally nonlinear, where the products stem from the fact that types recombine independently. This nonlinearity is the reason that an explicit solution cannot be given as before.

A notable exception is provided by recombination events that occur at links where one of the involved segments cannot have been affected by previous crossovers, namely the links $\frac{1}{2}$ and $\frac{2 n-1}{2}$. In this case, at least one of the factors in Eq. (19) becomes 1 (since, obviously, $G_{<\alpha}=\varnothing$ for $\alpha=\frac{1}{2}$ and $G_{>\alpha}=\varnothing$ for $\alpha=\frac{2 n-1}{2}$ ) and the resulting linear and triangular recursion can be solved. The coefficients for the corresponding link sets can be inferred directly (proof via simple induction) as

$$
\begin{aligned}
a_{\varnothing}(t) & =\eta^{t} \\
a_{\frac{1}{2}}(t) & =\left(\eta+\rho_{\frac{1}{2}}\right)^{t}-\eta^{t}, \\
a_{\frac{2 n-1}{2}}(t) & =\left(\eta+\rho_{\frac{2 n-1}{2}}\right)^{t}-\eta^{t}, \quad \text { and } \\
a_{\left\{\frac{1}{2}, \frac{2 n-1}{2}\right\}}(t) & =\eta^{t}-\left(\eta+\rho_{\frac{1}{2}}\right)^{t}-\left(\eta+\rho_{\frac{2 n-1}{2}}\right)^{t}+\left(\eta+\rho_{\frac{1}{2}}+\rho_{\frac{2 n-1}{2}}\right)^{t} .
\end{aligned}
$$

This explains the availability of an explicit solution for the model with up to three sites, where we do not have links other than $\frac{1}{2}$ and/or $\frac{3}{2}$, so that all corresponding coefficients can be determined explicitly. Indeed, one recovers (18) with $n=2$ and $\eta=1-\rho_{\frac{1}{2}}-\rho_{\frac{3}{2}}$.

So far, we have observed that the product structure of the coefficient functions, known from continuous time, is lost in discrete time from three sites onwards; this reflects the dependence of links. In contrast, the linearity of the iteration is only lost from four sites onwards. The latter can be understood further by comparison of (19) with the differential equations for the coefficients of the continuous-time model. These read:

$$
\frac{\mathrm{d}}{\mathrm{d} t} \widetilde{a}_{G}(t)=-\left(\sum_{\alpha \in L \backslash G} \tilde{\rho}_{\alpha}\right) \widetilde{a}_{G}(t)+\sum_{\alpha \in G} \tilde{\rho}_{\alpha} \widetilde{a}_{G \backslash\{\alpha\}}(t),
$$

that is, they are linear, with solution (9). Note that this linear dynamics emerges from a seemingly nonlinear one, namely the analogue of (19),

$$
\frac{\mathrm{d}}{\mathrm{d} t} \widetilde{a}_{G}(t)=-\left(\sum_{\alpha \in L} \tilde{\rho}_{\alpha}\right) \widetilde{a}_{G}(t)+\sum_{\alpha \in G} \tilde{\rho}_{\alpha}\left(\sum_{H \subseteq L} \tilde{a}_{G_{<\alpha} \cup H}(t)\right)\left(\sum_{K \subseteq L} \widetilde{a}_{K \cup G_{>\alpha}}(t)\right) .
$$

However, due to the product structure of the solution, the product term in the second sum, when inserting (9), reduces to a single term,

$$
\left(\sum_{H \subseteq L_{\geq \alpha}} \widetilde{a}_{G_{<\alpha} \cup H}(t)\right)\left(\sum_{K \subseteq L_{\leq \alpha}} \widetilde{a}_{K \cup G_{>\alpha}}(t)\right)=\widetilde{a}_{G}(t)+\widetilde{a}_{G \backslash\{\alpha\}}(t),
$$

which turns (22) into (21).

What happens here is the following. From four sites onwards (namely, beginning with $n=3$ and a crossover at $\alpha=\frac{3}{2}$, and both in discrete and continuous time), it happens that leading and trailing segments meet that both possess at least one link that may possibly have seen a previous cut. When 
a crossover at $\alpha$ takes place, the new joint distribution of cuts before and after $\alpha$ is formed as the product measure of the marginal distributions of cuts in the leading and trailing segments (cf. (19) and (22) - akin to the formation of product measures of marginal types by $R_{\alpha}$. In continuous time, the links are all independent, hence the new combination leaves the joint distribution of cuts unchanged. Therefore, a set $G$ of affected links (before and after $\alpha$ ) is simply augmented by $\alpha$ if $\alpha$ is a 'fresh' cut; this results in the linearity of (21). In discrete time, however, the dependence between the links, in particular between those in the leading and trailing segment, means that the formation of the product measure changes the joint distribution of affected links, in addition to the new cut at $\alpha$; thus (19) remains nonlinear.

Since we aim at an explicit solution of the discrete-time recombination model, we need to find a way to overcome the obstacles of nonlinearity. Inspired by the results of the continuous-time model, we now search for a transformation that decouples and linearises the dynamics.

To this end, we first investigate the behaviour of the $R_{G}$ and $T_{G}$ in the discrete-time model, since a deeper understanding of their actions will help us find a new transformation. We are still concerned with the LDE operators from the continuous-time model, because of their favourable structure and the existence of the inverse transformation (Möbius inversion). Moreover, as will become clear later, some of them still have the desired features and can be adopted directly for the discrete-time model. First, we need further notation.

Definition 1 Two links $\alpha, \beta \in L$ are called adjacent if $|\alpha-\beta|=1$. We say that a subset $\tilde{L} \subseteq L$ is contiguous if for any two links $\alpha, \beta \in \tilde{L}$ with $\alpha \leq \beta$, also all links between $\alpha$ and $\beta$ belong to $\tilde{L}$ (this includes the case $\tilde{L}=\varnothing)$. A non-empty contiguous set of links is written as $\tilde{L}=\left\{\ell_{\min }, \ldots, \ell_{\max }\right\}$.

Whereas, according to Theorem 2 all recombinators act linearly on the solution of the continuoustime recombination equation, this does not hold for the solution of the discrete-time model in general, though the following property still holds.

Lemma 1 Let $\left\{c_{G} \mid G \subseteq L\right\}$ be a family of non-negative numbers with $\sum_{G \subseteq L} c_{G}=1$. For an arbitrary $v \in \mathcal{P}(X)$ and for all $K \subseteq L$ with $L \backslash K$ contiguous, one has

$$
R_{K}\left(\sum_{G \subseteq L} c_{G} R_{G}(v)\right)=\sum_{G \subseteq L} c_{G} R_{G \cup K}(v) .
$$

Proof When $K=\varnothing$, the claim is clear, because $R_{\varnothing}=\mathbb{1}$ and $L$ itself is contiguous. Otherwise, we have $K=A \cup B$ with $A:=\left\{\frac{1}{2}, \frac{3}{2}, \ldots, \alpha\right\}$ and $B:=\left\{\beta, \beta+1, \ldots, \frac{2 n-1}{2}\right\}$ for some $\beta>\alpha$ (this includes the case $K=L$ via $\beta=\alpha+1)$. Since we work on $\mathcal{P}(X)$, we have $R_{K}=R_{B} R_{A}$ from Proposition 1 . With the projection $\pi_{i}: \mathcal{P}(X) \rightarrow \mathcal{P}\left(X_{i}\right)$ onto a single site $i$, we obtain

$$
\pi_{i} \cdot\left(\sum_{G \subseteq L} c_{G} R_{G}(v)\right)=\sum_{G \subseteq L} c_{G} \pi_{i} \cdot R_{G}(v)=\sum_{G \subseteq L} c_{G} \pi_{i} \cdot v=\pi_{i} \cdot v
$$

since $\pi_{i}$. is a linear operator and $\pi_{i}, R_{G}(v)=\pi_{i}, v$ by Proposition 1 For the contiguous set $A$ and $w:=\sum_{G \subseteq L} c_{G} R_{G}(v)$, we obtain, with the help of (23) and a repeated application of Proposition 1

$$
\begin{aligned}
& R_{A}\left(\sum_{G \subseteq L} c_{G} R_{G}(v)\right)=\pi_{0} \cdot w \otimes \cdots \otimes \pi_{\lfloor\alpha\rfloor} w \otimes \pi_{>\alpha} \cdot w \\
& =\pi_{0} \cdot v \otimes \cdots \otimes \pi_{\lfloor\alpha\rfloor} \cdot v \otimes \pi_{>\alpha} \cdot w=\sum_{G \subseteq L} c_{G}\left(\pi_{0} \cdot v \otimes \cdots \otimes \pi_{\lfloor\alpha\rfloor} \cdot v \otimes \pi_{>\alpha} \cdot R_{G}(v)\right) \\
& =\sum_{G \subseteq L} c_{G}\left(\pi_{0} \cdot R_{G}(v) \otimes \cdots \otimes \pi_{\lfloor\alpha\rfloor} \cdot R_{G}(v) \otimes \pi_{>\alpha} \cdot R_{G}(v)\right)=\sum_{G \subseteq L} c_{G} R_{A \cup G}(v) .
\end{aligned}
$$

An analogous calculation reveals $R_{B}\left(\sum_{G \subseteq L} c_{G} R_{A \cup G}(v)\right)=\sum_{G \subseteq L} c_{G} R_{A \cup B \cup G}(v)$ for contiguous $B$. This proves the assertion. 
The intuitive content of Lemma 1 falls into place with the explanation of Theorem 3 The linearity of the particular recombinators of Lemma 1 is due to the fact that $R_{K}$ produces only one segment, namely $L \backslash K$, that might be affected by previous recombination events while all other segments consist of only one site and thus cannot bring along cuts from 'the past'.

\section{Reduction to subsystems}

In this section, we show a certain product structure of the recombinators and the LDE operators. This will turn out as the key for constructing an appropriate transformation. Recall that a crossover at link $\alpha \in L$ partitions $S$ into $\{0, \ldots,\lfloor\alpha\rfloor\}$ and $\{\lceil\alpha\rceil, \ldots, n\}$. In general, recombination at the links belonging to $G=\left\{\alpha_{1}, \ldots, \alpha_{|G|}\right\} \subseteq L, \alpha_{1}<\alpha_{2}<\cdots<\alpha_{|G|}$, induces the following ordered partition $\mathcal{S}_{G}=\left\{J_{0}^{G}, J_{1}^{G}, \ldots, J_{|G|}^{G}\right\}$ of $S$ (see Fig. 11):

$$
J_{0}^{G}=\left\{0, \ldots,\left\lfloor\alpha_{1}\right\rfloor\right\}, J_{1}^{G}=\left\{\left\lceil\alpha_{1}\right\rceil, \ldots,\left\lfloor\alpha_{2}\right\rfloor\right\}, \ldots, J_{|G|}^{G}=\left\{\left\lceil\alpha_{|G|}\right\rceil, \ldots, n\right\} .
$$

Note that the partition is ordered due to the restriction to single crossovers. In connection with this, we have the sets of links that correspond to the respective parts of the partition $\mathcal{S}_{G}$ (Fig. 1). Namely, for $G=\left\{\alpha_{1}, \ldots, \alpha_{|G|}\right\} \subseteq L, \mathcal{L}_{G}:=\left\{I_{0}^{G}, I_{1}^{G}, \ldots, I_{|G|}^{G}\right\}$ with

$$
\begin{aligned}
& \qquad I_{0}^{G}=\left\{\alpha \in L: \frac{1}{2} \leq \alpha<\alpha_{1}\right\}, \quad I_{|G|}^{G}=\left\{\alpha \in L: \alpha_{|G|}<\alpha \leq \frac{2 n-1}{2}\right\}, \\
& \text { and } I_{\ell}^{G}=\left\{\alpha \in L: \alpha_{\ell}<\alpha<\alpha_{\ell+1}\right\} \text { for } 1 \leq \ell \leq|G|-1
\end{aligned}
$$

specifies the links belonging to the respective parts of $\mathcal{S}_{G}$ : the links associated with $J_{k}^{G} \in \mathcal{S}_{G}$, $0 \leq k \leq|G|$, are exactly those of $I_{k}^{G} \in \mathcal{L}_{G}$ (and vice versa).

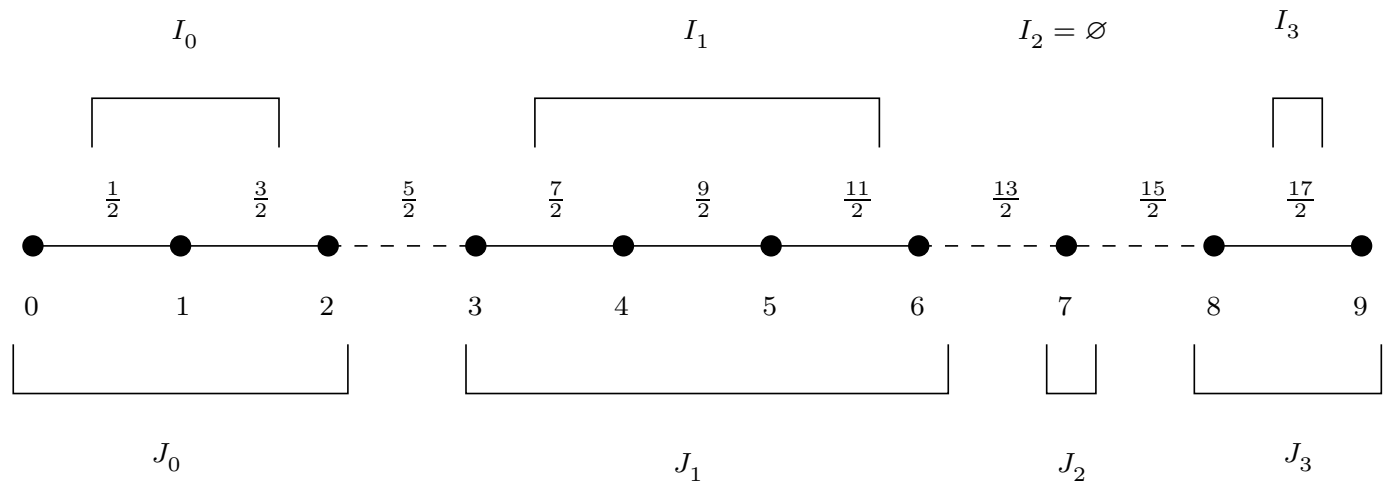

Fig. 1 A system with 10 sites (i.e., $S=\{0, \ldots, 9\}, L=\left\{\frac{1}{2}, \ldots, \frac{17}{2}\right\}$ ) cut at the links $G=\left\{\frac{5}{2}, \frac{13}{2}, \frac{15}{2}\right\}$ (broken lines). The resulting subsystems are $\mathcal{S}_{G}=\left\{J_{0}, \ldots, J_{3}\right\}$ and $\mathcal{L}_{G}=\left\{I_{0}, \ldots, I_{3}\right\}$ with $J_{0}=\{0,1,2\}, J_{1}=\{3,4,5,6\}$, $J_{2}=\{7\}$ and $J_{3}=\{8,9\}$ as well as $I_{0}=\left\{\frac{1}{2}, \frac{3}{2}\right\}, I_{1}=\left\{\frac{7}{2}, \frac{9}{2}, \frac{11}{2}\right\}, I_{2}=\varnothing$ and $I_{3}=\left\{\frac{17}{2}\right\}$ (the upper index $G$ is suppressed here for clarity).

With this definition, $I_{i}^{G}=\varnothing$ is possible for each $0 \leq i \leq|G|$ and will be included (possibly multiply) in $\mathcal{L}_{G}$. Furthermore, $\mathcal{L}_{\varnothing}:=\{L\}$, so that $I_{0}^{\varnothing}=L$. The upper index will be suppressed in cases where the corresponding set of links is obvious. Clearly, $\mathcal{L}_{G}$ is not a partition of $L$, whereas $\mathcal{S}_{G}$ is a partition of $S$.

This way, recombination at the links in $G \subseteq L$ produces several 'subsystems' (characterised through the sites $J_{k}$ and the corresponding links $I_{k}, 0 \leq k \leq|G|$ ) with respect to the 'full system' described through the sites $S$ and the links $L$. We demonstrate below that it is sufficient to consider 
these subsystems separately, a property that reduces the problem of dealing with the recombination dynamics. Note first that repeated application of (41) and (5) leads to

$$
\pi_{<\alpha} \cdot R_{G}^{(L)}(p)=R_{G_{<\alpha}}^{\left(L_{<\alpha}\right)}\left(\pi_{<\alpha} \cdot p\right) \quad \text { and } \quad \pi_{>\alpha} \cdot R_{G}^{(L)}(p)=R_{G_{>\alpha}}^{\left(L_{>\alpha}\right)}\left(\pi_{>\alpha} \cdot p\right)
$$

where $R_{G}^{(L)}$ is our usual recombinator acting on $\mathcal{P}(X)=\mathcal{P}\left(X_{0} \times \cdots \times X_{n}\right)$, and $R_{G_{<\alpha}}^{\left(L_{<\alpha}\right)}$ denotes the respective recombinator on $\mathcal{P}\left(X_{0} \times \cdots \times X_{\lfloor\alpha\rfloor}\right)$, which acts on the subsystem specified through the sites $L_{<\alpha}$ and cuts the links $G_{<\alpha}$ (and analogously for $R_{G_{>\alpha}}^{\left(L_{>\alpha}\right)}$ ). Likewise, recombinators $R_{H}^{\left(I_{i}\right)}$, $H \subseteq I_{i}$, acting on $\mathcal{P}\left(X_{J_{i}}\right)$, may be defined for all subsystems, $0 \leq i \leq|G|$, in the obvious way. For consistency, we define $R_{\varnothing}^{(\varnothing)}=\mathbb{1}$. From now on, the upper index specifies the corresponding system the $R_{G}$ (and, likewise, the $T_{G}$ ) are acting on. It will be suppressed in cases where the system is obvious. We now explain the inherent product structure of the recombinators:

Proposition 2 Let $G \subseteq L$. For each $\alpha \in G$ and $p \in \mathcal{P}(X)$, one has the identity

$$
R_{G}^{(L)}(p)=\left(R_{G_{<\alpha}}^{\left(L_{<\alpha}\right)}\left(\pi_{<\alpha} \cdot p\right)\right) \otimes\left(R_{G_{>\alpha}}^{\left(L_{>\alpha}\right)}\left(\pi_{>\alpha} \cdot p\right)\right) .
$$

Proof For $\alpha \in G$, Proposition 1 implies :

$$
\begin{aligned}
R_{G}^{(L)}(p) & =R_{\alpha}^{(L)}\left(R_{G}^{(L)}(p)\right)=\left(\pi_{<\alpha} \cdot R_{G}^{(L)}(p)\right) \otimes\left(\pi_{>\alpha} \cdot R_{G}^{(L)}(p)\right) \\
& =\left(R_{G_{<\alpha}}^{\left(L_{<\alpha}\right)}\left(\pi_{<\alpha} \cdot p\right)\right) \otimes\left(R_{G_{>\alpha}}^{\left(L_{>\alpha}\right)}\left(\pi_{>\alpha} \cdot p\right)\right),
\end{aligned}
$$

where the last step follows from (25).

This proposition carries over to the LDE operators:

Proposition 3 On $\mathcal{P}(X)$, the LDE operators satisfy

$$
T_{G}^{(L)}(p)=\left(T_{G_{<\alpha}}^{\left(L_{<\alpha}\right)}\left(\pi_{<\alpha} \cdot p\right)\right) \otimes\left(T_{G_{>\alpha}}^{\left(L_{>\alpha}\right)}\left(\pi_{>\alpha} \cdot p\right)\right) \quad \text { for all } \alpha \in G,
$$

where $T_{G_{<\alpha}}^{\left(L_{<\alpha}\right)}$ and $T_{G_{>\alpha}}^{\left(L_{>\alpha}\right)}$ now describe the operators acting on the simplices $\mathcal{P}\left(X_{0} \times \cdots \times X_{\lfloor\alpha\rfloor}\right)$ and $\mathcal{P}\left(X_{\lceil\alpha\rceil} \times \cdots \times X_{n}\right)$, respectively.

Proof Let $\alpha \in G$. Using the product structure from Proposition 2 and splitting the sum into two disjoint parts, one obtains

$$
\begin{aligned}
T_{G}^{(L)}(p)=\sum_{H \supseteq G}(-1)^{|H-G|} R_{H}^{(L)}(p)=\sum_{H \supseteq G}(-1)^{|H-G|}\left(\left(R_{H_{<\alpha}}^{\left(L_{<\alpha}\right)}\left(\pi_{<\alpha} \cdot p\right)\right) \otimes\left(R_{H_{>\alpha}}^{\left(L_{>\alpha}\right)}\left(\pi_{>\alpha} \cdot p\right)\right)\right) \\
=\sum_{L \backslash\{\alpha\} \supseteq H \supseteq G \backslash\{\alpha\}}(-1)^{|H-G \backslash\{\alpha\}|}\left(\left(R_{H_{<\alpha}}^{\left(L_{<\alpha}\right)}\left(\pi_{<\alpha} \cdot p\right)\right) \otimes\left(R_{H_{>\alpha}}^{\left(L_{>\alpha}\right)}\left(\pi_{>\alpha} \cdot p\right)\right)\right) \\
=\sum_{L_{<\alpha} \supseteq H_{<\alpha} \supseteq G_{<\alpha}}(-1)^{\left|H_{<\alpha}-G_{<\alpha}\right|} \sum_{L_{<\alpha} \supseteq H_{<\alpha} \supseteq G_{<\alpha}}(-1)^{\left|H_{>\alpha}-G_{>\alpha}\right|}\left(R_{H_{<\alpha}}^{\left(L_{<\alpha}\right)}\left(\pi_{<\alpha} \cdot p\right) \otimes R_{H_{>\alpha}}^{\left(L_{>\alpha}\right)}\left(\pi_{>\alpha} \cdot p\right)\right) \\
=\left(\sum_{L_{>\alpha} \supseteq H_{>\alpha} \supseteq G_{>\alpha}}\left(\sum_{L_{>\alpha} \supseteq H_{<\alpha} \mid}\left(R_{H_{<\alpha}}^{\left(L_{<\alpha}\right)}\left(\pi_{<\alpha} \cdot p\right)\right)\right)\right. \\
=\left(T_{G_{<\alpha}}^{\left(L_{<\alpha}\right)}\left(\pi_{<\alpha} p\right)\right) \otimes\left(T^{\left|H_{>\alpha}-G_{>\alpha}\right|}\left(R_{G_{>\alpha}}^{\left(L_{>\alpha}\right)}\left(\pi_{>\alpha} \cdot p\right)\right)\right) \\
\left.\quad\left(\pi_{>\alpha} \cdot p\right)\right),
\end{aligned}
$$

which establishes the claim. 
Using this argument iteratively on the respective segments, one easily obtains

$$
T_{G}^{(L)}(p)=\left(T_{\varnothing}^{\left(I_{0}\right)}\left(\pi_{J_{0}} . p\right)\right) \otimes\left(T_{\varnothing}^{\left(I_{1}\right)}\left(\pi_{J_{1}} \cdot p\right)\right) \otimes \cdots \otimes\left(T_{\varnothing}^{\left(I_{|G|}\right)}\left(\pi_{J_{|G|}} \cdot p\right)\right),
$$

where the upper index specifies the corresponding subsystems associated with $G$, compare (24). Hence, the effect of $T_{G}$ on the full system is given by that of $T_{\varnothing}$ on the respective subsystems corresponding to $G$.

Our goal is now to study the effect of the $R_{G}$ and $T_{G}$ on $\Phi$, the right-hand side of the recombination equation (15). This will show us in more detail when and why the LDE operators from the continuous-time model are not sufficient for solving the discrete-time model and, at the same time, will direct us to the new transformation.

If $\Phi^{(L)}$ denotes the right-hand side of the recombination equation on the full simplex $\mathcal{P}\left(X_{0} \times\right.$ $\left.\cdots \times X_{n}\right)$, then, for any contiguous $I=\{\alpha, \ldots, \beta\} \subseteq L$, the right-hand side of the recombination equation on the subsimplex $\mathcal{P}\left(X_{\lfloor\alpha\rfloor} \times \cdots \times X_{\lceil\beta\rceil}\right)$ will be denoted with $\Phi^{(I)}$. Again, we suppress the upper index when the simplex is obvious.

Proposition 4 For the right-hand side of the recombination equation,

$$
\Phi^{(L)}(p)=\eta p+\sum_{\alpha \in L} \rho_{\alpha} R_{\alpha}^{(L)}(p)=p+\sum_{\alpha \in L} \rho_{\alpha}\left(R_{\alpha}^{(L)}-\mathbb{1}^{(L)}\right)(p)
$$

one finds

$$
R_{\alpha}^{(L)}\left(\Phi^{(L)}(p)\right)=\left(\Phi^{\left(L_{<\alpha}\right)}\left(\pi_{<\alpha} \cdot p\right)\right) \otimes\left(\Phi^{\left(L_{>\alpha}\right)}\left(\pi_{>\alpha} \cdot p\right)\right)
$$

for every $\alpha \in L$ and $p \in \mathcal{P}(X)$.

Proof Since $R_{\alpha}^{(L)}\left(\Phi^{(L)}(p)\right)=\left(\pi_{<\alpha \cdot}\left(\Phi^{(L)}(p)\right)\right) \otimes\left(\pi_{>\alpha \cdot}\left(\Phi^{(L)}(p)\right)\right)$, we obtain with the help of (25):

$$
\begin{aligned}
& \pi_{<\alpha \cdot}\left(\Phi^{(L)}(p)\right)=\pi_{<\alpha} \cdot\left(p+\sum_{\beta \in L} \rho_{\beta}\left(R_{\beta}^{(L)}-\mathbb{1}^{(L)}\right)(p)\right) \\
& =\pi_{<\alpha} \cdot p+\sum_{\beta<\alpha} \rho_{\beta}\left(R_{\beta}^{\left(L_{<\alpha}\right)}-\mathbb{1}^{\left(L_{<\alpha}\right)}\right)\left(\pi_{<\alpha} \cdot p\right)+\sum_{\beta \geq \alpha} \rho_{\beta}\left(R_{\varnothing}^{\left(L_{<\alpha}\right)}-\mathbb{1}^{\left(L_{<\alpha}\right)}\right)\left(\pi_{<\alpha} \cdot p\right) \\
& =\pi_{<\alpha} \cdot p+\sum_{\beta<\alpha} \rho_{\beta}\left(R_{\beta}^{\left(L_{<\alpha}\right)}-\mathbb{1}^{\left(L_{<\alpha}\right)}\right)\left(\pi_{<\alpha} \cdot p\right)=\Phi^{\left(L_{<\alpha}\right)}\left(\pi_{<\alpha} \cdot p\right) .
\end{aligned}
$$

Analogously, one obtains $\pi_{>\alpha} \cdot\left(\Phi^{(L)}(p)\right)=\Phi^{\left(L_{>\alpha}\right)}\left(\pi_{>\alpha} \cdot p\right)$, and the assertion follows.

More generally, this theorem implies inductively that

$$
R_{G}^{(L)}\left(\Phi^{(L)}(p)\right)=\left(\Phi^{\left(I_{0}\right)}\left(\pi_{J_{0}} . p\right)\right) \otimes\left(\Phi^{\left(I_{1}\right)}\left(\pi_{J_{1}} \cdot p\right)\right) \otimes \cdots \otimes\left(\Phi^{\left(I_{|G|}\right)}\left(\pi_{J_{|G|} .} p\right)\right) .
$$

Finally, for the interaction between the $T_{G}^{(L)}$ and $\Phi^{(L)}$, we have the following result.

Proposition 5 For the LDE operators (10) and all $G \subseteq L$, one has

$$
T_{G}^{(L)}\left(\Phi^{(L)}(p)\right)=\left(T_{\varnothing}^{\left(I_{0}\right)}\left(\Phi^{\left(I_{0}\right)}\left(\pi_{J_{0}} \cdot p\right)\right)\right) \otimes\left(T_{\varnothing}^{\left(I_{1}\right)}\left(\Phi^{\left(I_{1}\right)}\left(\pi_{J_{1}} \cdot p\right)\right)\right) \otimes \cdots \otimes\left(T_{\varnothing}^{\left(I_{|G|}\right)}\left(\Phi^{\left(I_{|G|}\right)}\left(\pi_{J_{|G|}} \cdot p\right)\right)\right)
$$

with $I_{0}, \ldots, I_{|G|}$ according to (24).

Proof Using (27) and (26), one calculates

$$
\begin{aligned}
T_{G}^{(L)}\left(\Phi^{(L)}(p)\right) & =T_{G}^{(L)}\left(R_{G}^{(L)}\left(\Phi^{(L)}(p)\right)\right)=T_{G}^{(L)}\left(\left(\Phi^{\left(I_{0}\right)}\left(\pi_{J_{0}} . p\right)\right) \otimes \cdots \otimes\left(\Phi^{\left(I_{|G|}\right)}\left(\pi_{\left.J_{|G|} . p\right)}\right)\right)\right. \\
& =\left(T_{\varnothing}^{\left(I_{0}\right)}\left(\Phi^{\left(I_{0}\right)}\left(\pi_{J_{0}} . p\right)\right)\right) \otimes \cdots \otimes\left(T_{\varnothing}^{\left(I_{|G|}\right)}\left(\Phi^{\left(I_{|G|}\right)}\left(\pi_{J_{|G|}} p\right)\right)\right),
\end{aligned}
$$

which establishes the formula. 
This result is of particular significance since it shows that, to determine the effect of the $T_{G}^{(L)}$ on $\Phi$, it is sufficient to know the action of the $T_{\varnothing}$ on the subsystems that correspond to $G$. Hence, we now need to determine $T_{\varnothing} \circ \Phi$. It will turn out that this relies crucially on the commutators of $R_{G}$ with $\Phi$, which will be the subject of the next section.

\section{The commutator and linearisation}

The more algebraic approach of [2], which was later generalised by Popa [16], suggests to further analyse the problem in terms of commuting versus non-commuting quantities. For $G \subseteq L$, the commutator is defined as $\left[R_{G}, \Phi\right]:=R_{G} \circ \Phi-\Phi \circ R_{G}$. Recall that, in the continuous-time model, the linear action of the recombinators on the solution of the differential equations entails that the corresponding forward flow commutes with each recombinator (see Corollary 1). But this no longer holds for discrete time: $\left[R_{G}, \Phi\right]=0$ is not true in general. We are interested in the commutators because - as we will see in a moment - they lead us to the evaluation of $T_{\varnothing} \circ \Phi$, and this in turn gives $T_{G} \circ \Phi$ (see Proposition [5).

Proposition 6 Let $\eta=1-\sum_{\alpha \in L} \rho_{\alpha}$ as before. On $\mathcal{P}(X)$, one has

$$
T_{\varnothing} \circ \Phi=\eta T_{\varnothing}+\sum_{G \subseteq L}(-1)^{|G|}\left[R_{G}, \Phi\right] .
$$

Proof Expressing the left-hand side as

$$
T_{\varnothing} \circ \Phi=\sum_{G \subseteq L}(-1)^{|G|}\left(R_{G} \circ \Phi\right)=\sum_{G \subseteq L}(-1)^{|G|}\left(\Phi \circ R_{G}\right)+\sum_{G \subseteq L}(-1)^{|G|}\left[R_{G}, \Phi\right]
$$

and using $\Phi=\eta \mathbb{1}+\sum_{\alpha \in L} \rho_{\alpha} R_{\alpha}$, one calculates

$$
\begin{aligned}
\sum_{G \subseteq L}(-1)^{|G|}\left(\Phi \circ R_{G}\right) & =\sum_{\alpha \in L}\left(\sum_{G \subseteq L}(-1)^{|G|} \rho_{\alpha} R_{\alpha} R_{G}\right)+\eta \sum_{G \subseteq L}(-1)^{|G|} R_{G} \\
& =\eta T_{\varnothing}+\sum_{\alpha \in L} \sum_{\substack{G \subseteq L \\
\alpha \notin G}}\left((-1)^{|G|} \rho_{\alpha} R_{\alpha} R_{G}+(-1)^{|G \cup\{\alpha\}|} \rho_{\alpha} R_{G \cup\{\alpha\}}\right) \\
& =\eta T_{\varnothing}+\sum_{\alpha \in L}\left(\sum_{\substack{G \subseteq L \\
\alpha \notin G}}(-1)^{|G|} \rho_{\alpha}\left(R_{\alpha} R_{G}-R_{G \cup\{\alpha\}}\right)\right)=\eta T_{\varnothing},
\end{aligned}
$$

which shows the claim.

Proposition 6 shows that $T_{\varnothing}$ only yields a diagonal component if all recombinators commute with $\Phi$. We now need to determine the commutator $\left[R_{G}, \Phi\right]$. To this end, it is advantageous to introduce a new set of operators.

Definition 2 For $G \subseteq K \subseteq L$, we define the operators

$$
\widetilde{T}_{G, K}:=\sum_{G \subseteq H \subseteq K}(-1)^{|H-G|} R_{H} .
$$

Equivalently, for any $M \subseteq L \backslash G$, this means that

$$
\widetilde{T}_{G, G \cup M}=\sum_{G \subseteq H \subseteq G \cup M}(-1)^{|H-G|} R_{H}=\sum_{K \subseteq M}(-1)^{|K|} R_{G \cup \dot{ } K} .
$$


These operators act on the full simplex and can be interpreted in analogy to the original LDE operators (10), where the links in the complement of $G \dot{\cup} M$ (the disjoint union of $G$ and $M$ ) are regarded as inseparable. If necessary, we will specify the system the operators are acting on by an upper index as before.

Lemma 2 On $\mathcal{P}(X)$, the operators (28) satisfy

$$
\widetilde{T}_{G, G}=R_{G} \quad \text { and } \quad \widetilde{T}_{G, L}=T_{G}
$$

They have a product structure,

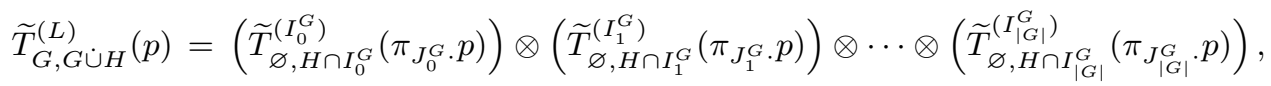

for all $H \subseteq L \backslash G$. Moreover, one has

$$
\widetilde{T}_{G, G \cup \dot{ } M}=\sum_{G \subseteq H \subseteq L \backslash M} T_{H}=\sum_{K \subseteq L \backslash(M \cup G)} T_{G \dot{\cup} K}
$$

for all $G, M \subseteq L$ with $G \cap M=\varnothing$. Consequently, Möbius inversion returns $T_{G}$ as

$$
T_{G}=\sum_{G \subseteq H \subseteq L \backslash M}(-1)^{|H-G|} \widetilde{T}_{H, H \cup M} .
$$

Proof The first assertion is obvious; the second is analogous to (26) and follows along the same lines. Relation (30) is true since

$$
\begin{aligned}
\widetilde{T}_{G, G \dot{\cup} M} & =\sum_{K \subseteq M}(-1)^{|K|} R_{G \dot{\cup} K}=\sum_{K \subseteq M}(-1)^{|K|} \sum_{H \supseteq G \cup \dot{ }} T_{H} \\
& =\sum_{H \supseteq G} \sum_{\substack{K \subseteq M \\
K \subseteq H}}(-1)^{|K|} T_{H}=\sum_{H \supseteq G} T_{H} \sum_{K \subseteq M \cap H}(-1)^{|K|} \\
& =\sum_{H \supseteq G} \delta_{M \cap H, \varnothing} T_{H}=\sum_{G \subseteq H \subseteq L \backslash M} T_{H} .
\end{aligned}
$$

In the second-last step, we used that, if $H$ is a finite set, one has

$$
\sum_{G \subseteq H}(-1)^{|G|}=\delta_{H, \varnothing}
$$

which is the key property of the Möbius function of ordered partitions.

Before we turn to the commutator, we introduce a new function, the separation function, which will allow for a clear and compact notation.

Definition 3 For $G, H \subseteq L$ with $G \cap H=\varnothing$, we say that $G$ separates $H$ if, for all $\alpha, \beta \in H$ with $\alpha<\beta$, there is a $\gamma \in G$ with $\alpha<\gamma<\beta$. Hence, we define the separation function as

$$
\operatorname{sep}(G, H)=\left\{\begin{array}{l}
1, \text { if } G \text { separates } H \\
0, \text { otherwise. }
\end{array}\right.
$$

In the particular cases $H=\varnothing$ and $H=\{\alpha\}, \alpha \in L$, we define $\operatorname{sep}(G, H)=1$ for all $G \subseteq L$, and it is understood that $\operatorname{sep}(G, H)=0$ whenever $G \cap H \neq \varnothing$.

First, let us summarise some elementary properties of the separation function.

Lemma 3 The separation function $\operatorname{sep}(G, H)$ with $H \subseteq L \backslash G$ has the following properties: 
1. $\operatorname{sep}(G, H)=0$, if $H$ contains any adjacent links;

2. $\operatorname{sep}(G, H)=0$ implies $\operatorname{sep}\left(G^{\prime}, H\right)=0$ for all $G^{\prime} \subseteq G$;

3. $\operatorname{sep}(G, H)=0$ whenever $L \backslash G$ is contiguous with $H \subseteq L \backslash G$ and $|H| \geq 2$;

4. $\operatorname{sep}(G, H)=1$ implies $I_{i}^{H} \cap G \neq \varnothing$ for all $i \in\{1, \ldots,|H|-1\}$.

Later, we need the following summation formula for the separation function.

Lemma 4 Let $H, K \subseteq L$ with $H \neq \varnothing, H \cap K=\varnothing$, and $I_{i}^{H}$ defined as in (24). Then

$$
\sum_{G \subseteq K}(-1)^{|G|} \operatorname{sep}(G, H)=\operatorname{sep}(K, H)(-1)^{|H|-1} \delta_{K \cap I_{0}^{H}, \varnothing} \delta_{K \cap I_{|H|}^{H}, \varnothing} .
$$

Proof For $\operatorname{sep}(K, H)=0$, the claim is clear by Lemma 3(2). We now define $A_{i}:=K \cap I_{i}^{H}$ for all $i \in\{0, \ldots,|H|\}$. Then, for $\operatorname{sep}(K, H)=1$, it follows from Lemma $3(4)$ that $A_{j} \neq \varnothing$ for all $1 \leq j \leq|H|-1$. Likewise, since $G \subseteq K, \operatorname{sep}(G, H)=1$ if and only if $G \cap I_{j}^{H} \neq \varnothing$ for all $1 \leq j \leq|H|-1$, with no condition emerging for $G \cap I_{0}^{H}$ or $G \cap I_{|H|}^{H}$. This gives

$$
\sum_{G \subseteq K}(-1)^{|G|} \operatorname{sep}(G, H)=\sum_{B_{0} \subseteq A_{0}}(-1)^{\left|B_{0}\right|} \prod_{i=1}^{|H|-1}\left(\sum_{\substack{B_{i} \subseteq A_{i} \\ B_{i} \neq \varnothing}}(-1)^{\left|B_{i}\right|}\right) \sum_{B_{|H|} \subseteq A_{|H|}}(-1)^{\left|B_{|H|}\right|}=\prod_{j=0}^{|H|} F_{j} .
$$

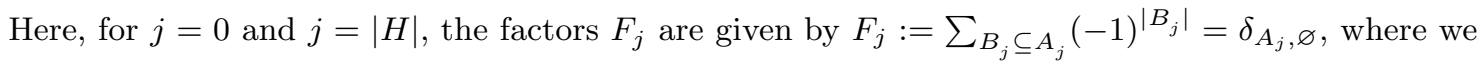
have used (32). For $1 \leq j \leq|H|-1$,

$$
F_{j}:=\sum_{\substack{B_{j} \subseteq A_{j} \\ B_{j} \neq \varnothing}}(-1)^{\left|B_{j}\right|}=-1+\sum_{B_{j} \subseteq A_{j}}(-1)^{\left|B_{j}\right|}=-1+\delta_{A_{j}, \varnothing}=-1,
$$

where we have again used (32) in the second-last step, and $A_{j} \neq \varnothing$ in the last.

With this notation, let us take a closer look at $R_{G}^{(L)}\left(\Phi^{(L)}(p)\right)$ for $G \subseteq L$. Evaluating (27) explicitly, using Definition 2, expanding and using the product structure (29) backwards gives

$$
\begin{aligned}
& R_{G}^{(L)}\left(\Phi^{(L)}(p)\right)=\left(\pi_{J_{0}} . p+\sum_{\alpha_{0} \in I_{0}} \rho_{\alpha_{0}}\left(R_{\alpha_{0}}^{\left(I_{0}\right)}-\mathbb{1}^{\left(I_{0}\right)}\right)\left(\pi_{J_{0}} . p\right)\right) \otimes \cdots \otimes
\end{aligned}
$$

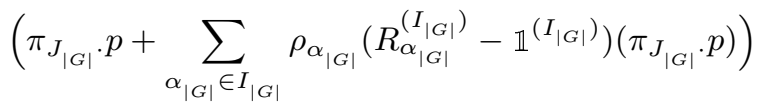

$$
\begin{aligned}
& =\left(\mathbb{1}^{\left(I_{0}\right)}-\sum_{\alpha_{0} \in I_{0}} \rho_{\alpha_{0}} \widetilde{T}_{\varnothing, \alpha_{0}}^{\left(I_{0}\right)}\right)\left(\pi_{J_{0}} \cdot p\right) \otimes \cdots \otimes\left(\mathbb{1}^{\left(I_{|G|}\right)}-\sum_{\alpha_{|G|} \in I_{|G|}} \rho_{\alpha_{|G|}} \widetilde{T}_{\varnothing, \alpha_{|G|}}^{\left(I_{0}\right)}\right)\left(\pi_{J_{|G|}} \cdot p\right) \\
& =\sum_{H \subseteq L \backslash G}(-1)^{|H|} \operatorname{sep}(G, H) \rho_{H} \widetilde{T}_{G, G \cup H}^{(L)}(p) \text {, }
\end{aligned}
$$

where, in the last step, we have further set $\rho_{H}=\prod_{\alpha \in H} \rho_{\alpha}$ for all $H \subseteq L$ (in particular, $\rho_{\varnothing}=1$ ) and used Lemma 3(4); note that the separation function is basically used as an indicator variable here. On the other hand, we obtain

$$
\begin{aligned}
\Phi \circ R_{G} & =\sum_{\alpha \in L \backslash G} \rho_{\alpha} R_{\alpha} R_{G}+\left(1-\sum_{\alpha \in L \backslash G} \rho_{\alpha}\right) R_{G} \\
& =\widetilde{T}_{G, G}-\sum_{\alpha \in L \backslash G} \rho_{\alpha} \widetilde{T}_{G, G \dot{\cup}\{\alpha\}} \\
& =\operatorname{sep}(G, \varnothing) \widetilde{T}_{G, G}-\sum_{\alpha \in L \backslash G} \operatorname{sep}(G,\{\alpha\}) \rho_{\alpha} \widetilde{T}_{G, G \dot{\cup}\{\alpha\}},
\end{aligned}
$$

which finally yields the commutator. 
Theorem 4 For all $G \subseteq L$, the commutator on $\mathcal{P}(X)$ is given by

$$
\left[R_{G}, \Phi\right]=\sum_{\substack{H \subseteq L \backslash G \\|\bar{H}| \geq 2}}(-1)^{|H|} \operatorname{sep}(G, H) \rho_{H} \widetilde{T}_{G, G \dot{\cup} H}
$$

Please note that, by the properties of the separation function, many of the summands vanish. In particular, $\left[R_{G}, \Phi\right]=0$ whenever $|L \backslash G| \leq 1$.

Corollary $2\left[R_{G}, \Phi\right]=0$ if $L \backslash G$ is contiguous.

Proof By Theorem 4. only terms with $|H| \geq 2$ need be considered. For these, Lemma 3) (3) tells us that $\operatorname{sep}(G, H)=0$ if $L \backslash G$ is contiguous and $H \subseteq L \backslash G$. Hence, $\left[R_{G}, \Phi\right]=0$.

Let us note in passing that the converse direction of Corollary 2 may fail if the site spaces are sufficiently trivial. Nevertheless, in the generic case, $\left[R_{G}, \Phi\right]=0 \operatorname{implies} \operatorname{sep}(G, H)=0$ for all $H \subseteq L \backslash G$ with $|H| \geq 2$, because the relevant terms then cannot cancel each other. We omit a more precise discussion of this point, because we do not need it later on.

Recalling that $\Phi^{t}$ is the discrete-time analogue of $\varphi_{t}$, we can consider Corollary 2 as what is left of Corollary 1 in discrete time. Hence, it becomes clear why the LDE operators (10) from the continuous-time model do not suffice to linearise and decouple the discrete-time dynamics.

We still aim at determining $T_{\varnothing} \circ \Phi$ according to Proposition 6 ] expressing the commutator $\left[R_{G}, \Phi\right]$ in terms of the $T_{G}$ (which are related to the $\widetilde{T}_{G, G \cup M}$ via (30)).

Theorem 5 On $\mathcal{P}(X)$, the operators $T_{G}=T_{G}^{(L)}$ and $\Phi=\Phi^{(L)}$ satisfy

$$
T_{G}^{(L)} \circ \Phi^{(L)}=\sum_{K \supseteq G} z^{(L)}(G, K) T_{K}^{(L)}
$$

for all $G \subseteq L$. The coefficients $z^{(L)}(\varnothing, K), K \subseteq L$, are given by

$$
z^{(L)}(\varnothing, \varnothing)=1-\sum_{\alpha \in L} \rho_{\alpha}
$$

and, for $K \neq \varnothing$, by

$$
z^{(L)}(\varnothing, K)=-\sum_{H \subseteq L \backslash K} \rho_{H} \operatorname{sep}(K, H)\left(1-\delta_{H \cap I_{0}^{K}, \varnothing}\right)\left(1-\delta_{H \cap I_{|K|}^{K}, \varnothing}\right) .
$$

For $K \supseteq G \neq \varnothing$, the coefficients are recursively determined by

$$
z^{(L)}(G, K)=z^{\left(I_{0}^{G}\right)}\left(\varnothing, K \cap I_{0}^{G}\right) \cdot \ldots \cdot z^{\left(I_{|G|}^{G}\right)}\left(\varnothing, K \cap I_{|G|}^{G}\right) .
$$

Proof Let us first prove the case $G=\varnothing$. According to Proposition 6, we have $T_{\varnothing} \circ \Phi=\eta T_{\varnothing}+$ $\sum_{G^{\prime} \subseteq L}(-1)^{\left|G^{\prime}\right|}\left[R_{G^{\prime}}, \Phi\right]$, where $\eta=z^{(L)}(\varnothing, \varnothing)$ by definition. Let us thus evaluate the last term. In the first step, we insert the commutator from Theorem 4 , we then use Definition 2 and change the order of summation to arrive at

$$
\begin{aligned}
\sum_{G^{\prime} \subseteq L}(-1)^{\left|G^{\prime}\right|}\left[R_{G^{\prime}}, \Phi\right] & =\sum_{G^{\prime} \subseteq L}(-1)^{\left|G^{\prime}\right|} \sum_{\substack{H \subseteq L \backslash G^{\prime} \\
|H| \geq 2}}(-1)^{|H|} \operatorname{sep}\left(G^{\prime}, H\right) \rho_{H} \widetilde{T}_{G^{\prime}, G^{\prime} \cup H} \\
& =\sum_{G^{\prime} \subseteq L}(-1)^{\left|G^{\prime}\right|} \sum_{\substack{H \subseteq L \backslash G^{\prime} \\
|H| \geq 2}}(-1)^{|H|} \operatorname{sep}\left(G^{\prime}, H\right) \rho_{H} \sum_{G^{\prime} \subseteq K \subseteq L \backslash H} T_{K} \\
& =\sum_{K \subseteq L} T_{K} \sum_{\substack{H \subseteq L \backslash K \\
|H| \geq 2}}(-1)^{|H|} \rho_{H} \sum_{G^{\prime} \subseteq K}(-1)^{\left|G^{\prime}\right|} \operatorname{sep}\left(G^{\prime}, H\right),
\end{aligned}
$$


which does not contain any term with $T_{\varnothing}$. We can now compare coefficients for $T_{K}$. Note first that, by (36), we only need to consider sets $H \subseteq L \backslash K$, that is, $H \cap K=\varnothing$. In this case, $\delta_{K \cap I_{0}^{H}, \varnothing}=1-\delta_{H \cap I_{0}^{K}, \varnothing}$ and $\delta_{K \cap I_{|H|}^{H}, \varnothing}=1-\delta_{H \cap I_{|K|}^{K}, \varnothing}$. This is true since $K \cap I_{0}^{H}=\varnothing(\neq \varnothing)$ implies that the smallest element in $H$ is smaller (larger) than the smallest element in $K$, thus $H \cap I_{0}^{K} \neq \varnothing(=\varnothing)$ (and vice versa for

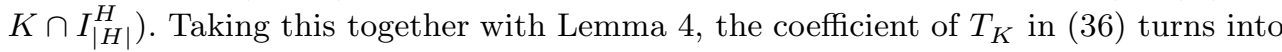

$$
\begin{aligned}
\sum_{\substack{H \subseteq L \backslash K \\
|H| \geq 2}}(-1)^{|H|} \rho_{H} & \sum_{G^{\prime} \subseteq K}(-1)^{\left|G^{\prime}\right|} \operatorname{sep}\left(G^{\prime}, H\right) \\
= & -\sum_{\substack{H \subseteq L \backslash K \\
|H| \geq 2}} \rho_{H} \operatorname{sep}(K, H)\left(1-\delta_{H \cap I_{0}^{K}, \varnothing}\right)\left(1-\delta_{H \cap I_{|K|}^{K}, \varnothing}\right) \\
& =-\sum_{H \subseteq L \backslash K} \rho_{H} \operatorname{sep}(K, H)\left(1-\delta_{H \cap I_{0}^{K}, \varnothing}\right)\left(1-\delta_{H \cap I_{|K|}^{K}, \varnothing}\right) .
\end{aligned}
$$

Note that, in the last step, the restriction on $|H|$ may be dropped since it is already implied by the factors involving the $\delta$-functions. This proves the claim for $G=\varnothing$. For the case $G \neq \varnothing$, we follow Proposition 5 and write, for $p \in \mathcal{P}(X)$,

$$
T_{G}^{(L)}\left(\Phi^{(L)}(p)\right)=\left(T_{\varnothing}^{\left(I_{0}\right)}\left(\Phi^{\left(I_{0}\right)}\left(\pi_{J_{0}} \cdot p\right)\right)\right) \otimes\left(T_{\varnothing}^{\left(I_{1}\right)}\left(\Phi^{\left(I_{1}\right)}\left(\pi_{J_{1}} \cdot p\right)\right)\right) \otimes \cdots \otimes\left(T_{\varnothing}^{\left(I_{|G|}\right)}\left(\Phi^{\left(I_{|G|}\right)}\left(\pi_{J_{|G|}} \cdot p\right)\right)\right) .
$$

Applying the above result for $G=\varnothing$ to each factor, and using the product structure of Proposition 5 backwards, establishes the claim.

Corollary 3 The coefficients $z(\varnothing, K)$ with $K \neq \varnothing$ can be expressed explicitly as

$$
z^{(L)}(\varnothing, K)=-\sum_{\alpha_{0} \in I_{0}^{K}} \rho_{\alpha_{0}}\left(\prod_{i=1}^{|K|-1}\left(1+\sum_{\alpha_{i} \in I_{i}^{K}} \rho_{\alpha_{i}}\right)\right) \sum_{\alpha_{|K|} \in I_{|K|}^{K}} \rho_{\alpha_{|K|}} .
$$

Proof Let us consider those $H$ whose contribution to the sum in (35) is not annihilated by the separation function or the $\delta$-functions. For $\operatorname{sep}(K, H)=1$ to hold, each $\alpha \in H$ must belong to a different $I_{i}^{K} \in \mathcal{L}_{K}$. Furthermore, $H$ must contain one element each from $I_{0}^{K}$ and $I_{|K|}^{K}\left(\alpha_{0}\right.$ and $\alpha_{|K|}$, respectively) to keep the factors involving the $\delta$-functions from vanishing. Thus, the sum in (35) may be factorised as claimed.

In particular, $z^{(L)}(\varnothing, K)=0$ if $K \cap\left\{\frac{1}{2}, \frac{2 n-1}{2}\right\} \neq \varnothing$. Taking this together with (34), one obtains $z^{(L)}(\varnothing, K)=\left(1-\sum_{\alpha \in L} \rho_{\alpha}\right) \delta_{K, \varnothing}$ for $K \subseteq L$ whenever $|L| \leq 2$, and hence, in these cases, $T_{\varnothing}^{(L)} \circ \Phi^{(L)}=$ $\left(1-\sum_{\alpha \in L} \rho_{\alpha}\right) T_{\varnothing}^{(L)}$ is already a diagonal component in line with the observation in Section 4 . Furthermore, Theorem [5 and (37) entail that $z^{(L)}(G, K)=0$ whenever

$$
K \cap\left(\bigcup_{0 \leq i \leq|G|}\left\{\min \left(I_{i}^{G}\right), \max \left(I_{i}^{G}\right)\right\}\right) \neq \varnothing .
$$

Theorem 5 reveals the linear structure inherent in the action of $T_{G}$ on $\Phi$. In fact, the structure is even triangular (with respect to the partial ordering) since $T_{G}^{(L)} \circ \Phi^{(L)}$ is a linear combination of the $T_{K}^{(L)}, K \supseteq G$. Thus, diagonalisation will boil down to recursive elimination. As a preparation, we make the following observation.

Corollary 4 If $L \neq \varnothing$, one has the relation $z^{(L)}(G, L)=0$ for all $\varnothing \subseteq G \subsetneq L$.

Proof When $\varnothing \subseteq G \subsetneq L$, the intersection in (38), with $K=L$, can never be empty, so that $z^{(L)}(G, L)=0$ follows. 


\section{Diagonalisation}

Motivated by the triangular structure of (33), we make the ansatz to define new operators $U_{G}$, $G \subseteq L$, as the following linear combination of the well-known $T_{G}$ :

$$
U_{G}=\sum_{H \supseteq G} c(G, H) T_{H}
$$

where the coefficients $c(G, H)$ are to be determined in such a way that they transform the recombination equation into a decoupled diagonal system, more precisely so that

$$
U_{G} \circ \Phi=\lambda_{G} U_{G}, \quad G \subseteq L
$$

with eigenvalues $\lambda_{G}$ that are still unknown as well. An example for this transformation can be found in Appendix A. Note first that, with the help of (33), Eqs. (39) and (40) may be rewritten as

$$
\begin{aligned}
U_{G} \circ \Phi= & c(G, G) T_{G} \circ \Phi+\sum_{N \supsetneq G} c(G, N)\left(T_{N} \circ \Phi\right) \\
= & c(G, G)\left(z^{(L)}(G, G) T_{G}+\sum_{K \supsetneq G} z^{(L)}(G, K) T_{K}\right) \\
& +\sum_{N \supsetneq G} c(G, N)\left(z^{(L)}(N, N) T_{N}+\sum_{M \supsetneq N} z^{(L)}(N, M) T_{M}\right) \\
& \stackrel{!}{=} \lambda_{G}\left(c(G, G) T_{G}+\sum_{N \supsetneq G} c(G, N) T_{N}\right)=\lambda_{G} U_{G} .
\end{aligned}
$$

Obviously, there is some freedom in the choice of the $c(G, G)$; we set $c(G, G)=1$ for all $G \subseteq L$ (and we will see shortly that this is consistent). Eq. (41) has the structure of an eigenvalue problem of a triangular matrix with coefficients $z^{(L)}(G, H)$, where the role of the unit vectors is taken by the $T_{H}$, and the $c(G, H), H \supseteq G$, take the roles of the components of the eigenvector corresponding to $\lambda_{G}$ (note that, by considering $c(G, H)$ for $H \supseteq G$ only, we have already exploited the triangular structure). Recall next that the eigenvalues of a triangular matrix are given by its diagonal entries, which are

$$
\lambda_{G}=z^{(L)}(G, G)=\prod_{i=0}^{|G|} z^{\left(I_{i}^{G}\right)}(\varnothing, \varnothing)=\prod_{i=0}^{|G|}\left(1-\sum_{\alpha_{i} \in I_{i}^{G}} \rho_{\alpha_{i}}\right)
$$

by Theorem [5] In particular, $\lambda_{\varnothing}=\eta=1-\sum_{\alpha \in L} \rho_{\alpha} \geq 0$. The $\lambda_{G}$ describe the probability that there is no further recombination between the respective sites of the subsystems corresponding to $G$; they have already been identified by Bennett [5] and Dawson [9, 10].

Lemma 5 For all $G, H \subseteq L$ with $G \subsetneq H$, one has $\lambda_{G}<\lambda_{H}$.

Proof Let $\varnothing \subsetneq G \subsetneq L$. Then, for $H=G \dot{\cup}\{\beta\}$, with $\beta \in I_{i}^{G}$ for an arbitrary $i \in\{0, \ldots,|G|\}$, we see from (42) that $z^{(L)}(H, H)=\lambda_{H}$ and hence obtain

$$
\begin{aligned}
\lambda_{H} & =\left(\prod_{j=0}^{i-1}\left(1-\sum_{\alpha_{j} \in I_{j}^{G}} \rho_{\alpha_{j}}\right)\right)\left(1-\sum_{\substack{\alpha_{i} \in I_{i}^{G} \\
\alpha_{i}<\beta}} \rho_{\alpha_{i}}\right)\left(1-\sum_{\substack{\alpha_{i} \in I_{i}^{G} \\
\alpha_{i}>\beta}} \rho_{\alpha_{i}}\right)\left(\prod_{j=i+1}^{|G|}\left(1-\sum_{\alpha_{j} \in I_{j}^{G}} \rho_{\alpha_{j}}\right)\right) \\
& =\lambda_{G} \frac{\left(1-\sum_{\substack{\alpha_{i} \in I_{i}^{G} \\
\alpha_{i}<\beta}} \rho_{\alpha_{i}}\right)\left(1-\sum_{\substack{\alpha_{i} \in I_{i}^{G} \\
\alpha_{i}>\beta}} \rho_{\alpha_{i}}\right)}{\left(1-\sum_{\alpha_{i} \in I_{i}^{G}} \rho_{\alpha_{i}}\right)}>\lambda_{G},
\end{aligned}
$$


because all $\rho_{\alpha}$ are positive, as are all three terms in parentheses of the fraction, and $\rho_{\beta}>0$ by assumption. Finally, the argument also works for $\lambda_{\varnothing}=\eta$, provided $\eta>0$. Since $\lambda_{G}>0$ for all $G \neq \varnothing$, the claim trivially also holds for $\eta=0$. The assertion then follows inductively for any $H \supsetneq G$.

The coefficients $c(G, H)$ can now be calculated recursively as follows.

Theorem 6 The coefficients $c(G, H)$ of (39) are determined by $c(G, G)=1$ and

$$
c(G, H)=\frac{\sum_{H \supsetneq K \supseteq G} c(G, K) z^{(L)}(K, H)}{\lambda_{G}-\lambda_{H}}
$$

for $H \supsetneq G$. The coefficients of the inverse transformation of (39),

$$
T_{G}=\sum_{H \supseteq G} c^{*}(G, H) U_{H}, \quad \text { with } G \subseteq L,
$$

are determined by

$$
c^{*}(G, K)=-\sum_{K \supsetneq H \supseteq G} c^{*}(G, H) c(H, K),
$$

for $K \supsetneq G$ together with $c^{*}(G, G)=1$.

Proof Considering (41) with $c(G, G)=1$, comparing coefficients for $T_{H}, H \supsetneq G$, and observing (42), one obtains

$$
z^{(L)}(G, H)+c(G, H) \lambda_{H}+\sum_{H \supsetneq K \supsetneq G} c(G, K) z^{(L)}(K, H) \stackrel{!}{=} \lambda_{G} c(G, H)
$$

and the recursion for $c(G, H)$ follows. It is always well-defined for all $H \supsetneq G$, since $\lambda_{G}<\lambda_{H}$ by Lemma 5. The recursion for the coefficients of the inverse transformation follows directly from

$$
T_{G}=\sum_{H \supseteq G} c^{*}(G, H) U_{H}=\sum_{H \supseteq G} c^{*}(G, H) \sum_{K \supseteq H} c(H, K) T_{K}=\sum_{K \supseteq G} T_{K} \sum_{K \supseteq H \supseteq G} c^{*}(G, H) c(H, K)
$$

which enforces $\sum_{K \supseteq H \supseteq G} c^{*}(G, H) c(H, K)=\delta_{K, G}$, as the $T_{K}$ are distinct.

We now identify those $T_{G}$ that already give diagonal components of the discrete-time system:

Theorem 7 For all $G \subseteq L$ that satisfy $\left|I_{i}^{G}\right| \leq 2$ for all $i \in\{0, \ldots,|G|\}$, one has

$$
T_{G}(\Phi(p))=\lambda_{G} T_{G}(p)
$$

for $p \in \mathcal{P}(X)$.

Proof In this case, we have (38) for all $K \supsetneq G$, hence $z(G, K)=\lambda_{G} \delta_{K, G}$, from which the assertion follows via Theorem 5 .

Note that $\left|I_{i}^{G}\right| \leq 2$ for all $I_{i}^{G} \in \mathcal{L}_{G}$ simply implies that each subsystem consists of at most three sites, hence all subsystems can be reduced to the simple cases considered in Section 4. Then, for such $G, c(G, H)=c^{*}(G, H)=\delta_{G, H}$ for all $H \supseteq G$.

With the help of this transformation, we can finally specify the solution $p_{t}$ of the recombination equation in terms of the initial condition $p_{0}$. To this end, we first use the transformation (11) from the recombinators to the $T_{G}$ operators, and then relation (44) to arrive at the $U_{H}$ operators, which 
finally diagonalise the system according to (40). Finally, we use the appropriate inversions to return to the recombinators:

$$
\begin{aligned}
p_{t} & =\Phi^{t}\left(p_{0}\right)=R_{\varnothing}\left(\Phi^{t}\left(p_{0}\right)\right)=\sum_{G \subseteq L} T_{G}\left(\Phi^{t}\left(p_{0}\right)\right)=\sum_{G \subseteq L} \sum_{H \supseteq G} c^{*}(G, H) U_{H}\left(\Phi^{t}\left(p_{0}\right)\right) \\
& =\sum_{G \subseteq L} \sum_{H \supseteq G} c^{*}(G, H) \lambda_{H}^{t} U_{H}\left(p_{0}\right)=\sum_{G \subseteq L} \sum_{H \supseteq G} c^{*}(G, H) \lambda_{H}^{t} \sum_{M \supseteq H} c(H, M) T_{M}\left(p_{0}\right) \\
& =\sum_{G \subseteq L} \sum_{H \supseteq G} c^{*}(G, H) \lambda_{H}^{t} \sum_{M \supseteq H} c(H, M) \sum_{T \supseteq M}(-1)^{|T-M|} R_{T}\left(p_{0}\right) .
\end{aligned}
$$

The coefficient functions can now be extracted as follows.

Theorem 8 The coefficient functions $a_{G}(t)$ of the solution (15) of the recombination equation in discrete time may be expressed as

$$
a_{G}(t)=\sum_{M \subseteq G}(-1)^{|G-M|} \sum_{H \subseteq M} \sum_{K \subseteq H} c(H, M) \lambda_{H}^{t} c^{*}(K, H)
$$

for all $G \subseteq L$. Here, $c(H, M)$ and $c^{*}(K, H)$ are the coefficients of Theorem $[$.

To derive the asymptotic behaviour for large iteration numbers, we need the following property of the coefficients.

Lemma 6 The coefficients $c(G, L)$ and $c^{*}(G, L)$ satisfy $c(G, L)=c^{*}(G, L)=\delta_{G, L}$ for arbitrary $\varnothing \subseteq G \subseteq L$.

Proof We have $c(G, G)=c^{*}(G, G)=1$ for all $G$ by Theorem [6. The claim for $c(G, L)$ now follows from the recursion (43) together with Corollary 4 Inserting this into recursion (45) establishes the relation for the $c^{*}(G, L)$.

As an example, the path to a solution via the above chain of transformations for the model with five sites will be presented in Appendix A.

Finally, let us consider what happens in the limit as $t \rightarrow \infty$.

Proposition 7 The solution $p_{t}$ of the recombination equation (14) with initial condition $p_{0}$ satisfies

$$
p_{t} \stackrel{t \rightarrow \infty}{\longrightarrow} R_{L}\left(p_{0}\right)=\bigotimes_{i=0}^{n}\left(\pi_{i} \cdot p_{0}\right),
$$

with exponentially fast convergence in the norm topology.

Proof When expressing $p_{t}$ in terms of $U_{H}$ according to (46), we first observe $p_{t}=U_{L}\left(p_{0}\right)+$ $\sum_{G \subsetneq L} \sum_{H \supseteq G} c^{*}(G, H) \lambda_{H}^{t} U_{H}\left(p_{0}\right)$, because $\lambda_{L}=1$ and $c^{*}(G, L)=\delta_{G, L}$ by Lemma 6. Since $U_{L}=R_{L}$, we obtain the following estimate in the variation norm

$$
\begin{aligned}
\left\|p_{t}-R_{L}\left(p_{0}\right)\right\| & =\left\|\sum_{G \subsetneq L} \sum_{H \supseteq G} c^{*}(G, H) \lambda_{H}^{t} U_{H}\left(p_{0}\right)\right\| \\
& \leq \sum_{H \subsetneq L} \lambda_{H}^{t}\left\|\sum_{G \subseteq H} c^{*}(G, H) U_{H}\left(p_{0}\right)\right\| \stackrel{t \rightarrow \infty}{\longrightarrow} 0,
\end{aligned}
$$

which establishes the claim since $\lambda_{H}<1$ for $H \neq L$.

As was to be expected, the solution of the recombination equation converges towards the independent combination of the alleles, that is towards linkage equilibrium. 


\section{Discussion}

In this paper, we have investigated the dynamics of an 'infinite' population that evolves due to recombination alone. To this end, we assumed discrete (non-overlapping) generations, and restricted ourselves to the case of single crossovers. Previous results had shown that the corresponding singlecrossover dynamics in continuous time admits a closed solution [3]. This astonishing result is concordant with a 'hidden' linearity in the system that is due to independence of links. The fact that crossovers at different links occur independently manifests itself in the product structure of the coefficient functions of the solution ensuing from the linear action of the nonlinear recombination operators along the solution of the recombination equation. Additionally, in [3], a certain set of linkage disequilibria was found that linearise and diagonalise the dynamics.

Since the overwhelming part of the literature deals with discrete-time models, our aim was to find out whether, and to what extent, these continuous-time results carry over to single-crossover dynamics in discrete time. We could show that the discrete-time dynamics is far more complex than the continuous-time one, and, as a consequence, a closed solution cannot be given.

The main reason for these difficulties lies in the fact that the key feature of the continuous-time model, the independence of links, does not carry over to discrete time. This is due to interference: The occurrence of a recombination event in the discrete-time model forbids any further crossovers in the same generation. In connection with this, the recombinators do not, in general, act linearly on the right-hand side of the recombination equation. Likewise, the coefficient functions of the solution follow a nonlinear iteration that cannot be solved explicitly.

While Geiringer [11] developed a skilful procedure for the generation-wise evaluation of these coefficients, we constructed a method that allows for an explicit formula valid for all times, once the coefficients of the transformation have been determined recursively for a given system.

As in previous approaches, this is achieved by a transformation of the nonlinear, coupled system of equations to a linear diagonal one. This was done before by Bennett [5] and Dawson [9, 10] for the more general recombination equation (without restriction to single crossovers), and they presented an appropriate transformation that includes parameters that must be determined recursively. Unfortunately, the corresponding derivations are rather technical and fail to reveal the underlying mathematical structure. It was our aim to improve on this and add some structural insight. Unlike the previous approaches, we proceeded in two steps: first linearisation followed by diagonalisation. More precisely, it turns out that the LDE operators $T_{G}$, which both linearise and diagonalise the continuous-time system, still linearise the discrete-time dynamics, but fail to diagonalise it for four or more loci. However, the resulting linear system may be diagonalised in a second step. This relies on linear combinations $U_{G}$ of the $T_{G}$, with coefficients derived in a recursive manner.

As it must be, the transformation agrees with the one of Dawson [9, 10] when translated into his framework. (Note that our $c(G, H)$ are coefficients of $T_{H}$, whereas his coefficients belong to components of $R_{H}(p)$. Note also that SCR does not belong to the singular cases he excludes). It remains an interesting open problem how much of the above findings can be transferred to the general recombination model (i.e. without the restriction to single crossovers), where one loses the simplifying structure of ordered partitions.

Acknowledgements It is our pleasure to thank Th. Hustedt for critically reading the manuscript and K. Schneider for valuable suggestions to further improve it. This work was supported by DFG (Research training group Bioinformatics, and Dutch-German Bilateral Research Group on Mathematics of Random Spatial Models in Physics and Biology). 


\section{Appendix A: Five Sites}

To illustrate the construction, let us spell out the example of five sites. We have $S=\{0,1,2,3,4\}$ and $L=\left\{\frac{1}{2}, \frac{3}{2}, \frac{5}{2}, \frac{7}{2}\right\}$, the corresponding recombination probabilities $\rho_{\alpha}, \alpha \in L, \eta=1-\rho_{\frac{1}{2}}-\rho_{\frac{3}{2}}-\rho_{\frac{5}{2}}-\rho_{\frac{7}{2}}$, and a given initial population $p_{0}$. Aiming at determining the coefficient functions $a_{G}(t)$ for all $G \subseteq L$, we can immediately write down $a_{\varnothing}(t)=\eta^{t}, a_{\frac{1}{2}}(t)=\left(\eta+\rho_{\frac{1}{2}}\right)^{t}-\eta^{t}, a_{\frac{7}{2}}(t)=\left(\eta+\rho_{\frac{7}{2}}\right)^{t}-\eta^{t}$ and $a_{\left\{\frac{1}{2}, \frac{7}{2}\right\}}(t)=\eta^{t}-\left(\eta+\rho_{\frac{1}{2}}\right)^{t}-\left(\eta+\rho_{\frac{7}{2}}\right)^{t}+\left(\eta+\rho_{\frac{1}{2}}+\rho_{\frac{7}{2}}\right)^{t}$, see (20).

If we wanted to determine the remaining coefficient functions $a_{G}(t)$ for a given time $t$, they could be calculated using the method of Geiringer [11] (i.e. Theorem 31). But since we aim at a closed solution for all $t$, we use the procedure developed above. To determine the coefficients of Theorem 8 . we have to calculate the corresponding $c(G, H)$ and $c^{*}(G, H)$. Theorem 6 and 7 imply $U_{L}=T_{L}$, $U_{L \backslash\{\alpha\}}=T_{L \backslash\{\alpha\}}$ for all $\alpha \in L, U_{L \backslash\{\alpha, \beta\}}=T_{L \backslash\{\alpha, \beta\}}$ for all $\alpha, \beta \in L$, as well as $U_{\frac{3}{2}}=T_{\frac{3}{2}}$ and $U_{\frac{5}{2}}=T_{\frac{5}{2}}$. Hence, in these cases, the only non-vanishing coefficients are $c(L, L)=c(L \backslash\{\alpha\}, L \backslash\{\alpha\})=$ $c(L \backslash\{\alpha, \beta\}, L \backslash\{\alpha, \beta\})=c\left(\left\{\frac{3}{2}\right\},\left\{\frac{3}{2}\right\}\right)=c\left(\left\{\frac{5}{2}\right\},\left\{\frac{5}{2}\right\}\right)=1$ for all $\alpha, \beta \in L$. It remains to determine $U_{\frac{1}{2}}, U_{\frac{7}{2}}$ and $U_{\varnothing}$.

\section{Constructing $U_{\frac{1}{2}}$ :}

The recursion starts with $c\left(\left\{\frac{1}{2}\right\},\left\{\frac{1}{2}\right\}\right)=1$. Following (38), $z^{(L)}\left(\left\{\frac{1}{2}\right\}, H\right)=0$ for all $H \supsetneq\left\{\frac{1}{2}\right\}$ except for $H=\left\{\frac{1}{2}, \frac{5}{2}\right\}$, and thus the only non-zero $c\left(\left\{\frac{1}{2}\right\}, H\right), H \supsetneq\left\{\frac{1}{2}\right\}$, is

$$
c\left(\left\{\frac{1}{2}\right\},\left\{\frac{1}{2}, \frac{5}{2}\right\}\right)=\frac{z\left(\left\{\frac{1}{2}\right\},\left\{\frac{1}{2}, \frac{5}{2}\right\}\right)}{\lambda_{\frac{1}{2}}-\lambda_{\left\{\frac{1}{2}, \frac{5}{2}\right\}}}=\frac{\rho_{\frac{3}{2}} \rho_{\frac{7}{2}}}{\rho_{\frac{5}{2}}+\rho_{\frac{3}{2}} \rho_{\frac{7}{2}}}
$$

where we have used the recursion (43) and $\lambda_{\frac{1}{2}}=1-\rho_{\frac{3}{2}}-\rho_{\frac{5}{2}}-\rho_{\frac{7}{2}}, \lambda_{\left\{\frac{1}{2}, \frac{5}{2}\right\}}=\left(1-\rho_{\frac{3}{2}}\right)\left(1-\rho_{\frac{7}{2}}\right)$. So, for the transformation (39) we obtain

$$
U_{\frac{1}{2}}=T_{\frac{1}{2}}+\frac{\rho_{\frac{3}{2}} \rho_{\frac{7}{2}}}{\rho_{\frac{5}{2}}+\rho_{\frac{3}{2}} \rho_{\frac{7}{2}}} T_{\left\{\frac{1}{2}, \frac{5}{2}\right\}}
$$

so that $U_{\frac{1}{2}} \circ \Phi=\left(1-\rho_{\frac{3}{2}}-\rho_{\frac{5}{2}}-\rho_{\frac{7}{2}}\right) U_{\frac{1}{2}}$. Analogously,

$$
U_{\frac{7}{2}}=T_{\frac{7}{2}}+\frac{\rho_{\frac{1}{2}} \rho_{\frac{5}{2}}}{\rho_{\frac{3}{2}}+\rho_{\frac{1}{2}} \rho_{\frac{5}{2}}} T_{\left\{\frac{3}{2}, \frac{7}{2}\right\}}
$$

2. Constructing $U_{\varnothing}$ :

By (38), the only non-vanishing coefficients are $c(\varnothing, \varnothing), c\left(\varnothing,\left\{\frac{3}{2}\right\}\right), c\left(\varnothing,\left\{\frac{5}{2}\right\}\right)$, and $c\left(\varnothing,\left\{\frac{3}{2}, \frac{5}{2}\right\}\right)$. They are determined by the recursion (43) and lead to the following transformation (39):

$$
U_{\varnothing}=T_{\varnothing}+\frac{\rho_{\frac{1}{2}}\left(\rho_{\frac{5}{2}}+\rho_{\frac{7}{2}}\right)}{\rho_{\frac{3}{2}}+\rho_{\frac{1}{2}}\left(\rho_{\frac{5}{2}}+\rho_{\frac{7}{2}}\right)} T_{\frac{3}{2}}+\frac{\left(\rho_{\frac{1}{2}}+\rho_{\frac{3}{2}}\right) \rho_{\frac{7}{2}}}{\rho_{\frac{5}{2}}+\left(\rho_{\frac{1}{2}}+\rho_{\frac{3}{2}}\right) \rho_{\frac{7}{2}}} T_{\frac{5}{2}}+\frac{\rho_{\frac{1}{2}} \rho_{\frac{7}{2}}}{\rho_{\frac{1}{2}} \rho_{\frac{7}{2}}+\rho_{\frac{3}{2}}+\rho_{\frac{5}{2}}} T_{\left\{\frac{3}{2}, \frac{5}{2}\right\}} .
$$


Now that we know the $c(G, H)$, the coefficients $c^{*}(G, H)$ are calculated via (45). Finally, the remaining coefficient functions follow from Theorem 8

$$
\begin{aligned}
& a_{\frac{3}{2}}(t)=\frac{\rho_{\frac{3}{2}}}{\rho_{\frac{1}{2}}\left(\rho_{\frac{5}{2}}+\rho_{\frac{7}{2}}\right)+\rho_{\frac{3}{2}}}\left(\lambda_{\frac{3}{2}}^{t}-\lambda_{\varnothing}^{t}\right) \\
& a_{\frac{5}{2}}(t)=\frac{\rho_{\frac{5}{2}}}{\rho_{\frac{7}{2}}\left(\rho_{\frac{1}{2}}+\rho_{\frac{3}{2}}\right)+\rho_{\frac{5}{2}}}\left(\lambda_{\frac{5}{2}}^{t}-\lambda_{\varnothing}^{t}\right) \\
& a_{\left\{\frac{1}{2}, \frac{3}{2}\right\}}(t)=\lambda_{\left\{\frac{1}{2}, \frac{3}{2}\right\}}^{t}-\lambda_{\frac{1}{2}}^{t}-\frac{\rho_{\frac{3}{2}}}{\rho_{\frac{1}{2}}\left(\rho_{\frac{5}{2}}+\rho_{\frac{7}{2}}\right)+\rho_{\frac{3}{2}}}\left(\lambda_{\frac{3}{2}}^{t}-\lambda_{\varnothing}^{t}\right) \\
& a_{\left\{\frac{1}{2}, \frac{5}{2}\right\}}(t)=\frac{\rho_{\frac{5}{2}}}{\rho_{\frac{3}{2}} \rho_{\frac{7}{2}}+\rho_{\frac{5}{2}}}\left(\lambda_{\left\{\frac{1}{2}, \frac{5}{2}\right\}}^{t}-\lambda_{\frac{1}{2}}^{t}\right)-\frac{\rho_{\frac{5}{2}}}{\rho_{\frac{7}{2}}\left(\rho_{\frac{1}{2}}+\rho_{\frac{3}{2}}\right)+\rho_{\frac{5}{2}}}\left(\lambda_{\frac{5}{2}}^{t}-\lambda_{\varnothing}^{t}\right) \\
& a_{\left\{\frac{3}{2}, \frac{5}{2}\right\}}(t)=\frac{\rho_{\frac{3}{2}}+\rho_{\frac{5}{2}}}{\rho_{\frac{1}{2}} \rho_{\frac{7}{2}}+\rho_{\frac{3}{2}}+\rho_{\frac{5}{2}}} \lambda_{\left\{\frac{3}{2}, \frac{5}{2}\right\}}^{t}-\frac{\rho_{\frac{3}{2}}}{\rho_{\frac{1}{2}}\left(\rho_{\frac{5}{2}}+\rho_{\frac{7}{2}}\right)+\rho_{\frac{3}{2}}} \lambda_{\frac{3}{2}}^{t}-\frac{\rho_{\frac{5}{2}}}{\rho_{\frac{7}{2}}\left(\rho_{\frac{1}{2}}+\rho_{\frac{3}{2}}\right)+\rho_{\frac{5}{2}}} \lambda_{\frac{5}{2}}^{t} \\
& +\left(1-\frac{\left(\rho_{\frac{1}{2}}+\rho_{\frac{3}{2}}\right) \rho_{\frac{7}{2}}}{\rho_{\frac{7}{2}}\left(\rho_{\frac{1}{2}}+\rho_{\frac{3}{2}}\right)+\rho_{\frac{5}{2}}}-\frac{\left(\rho_{\frac{5}{2}}+\rho_{\frac{7}{2}}\right) \rho_{\frac{1}{2}}}{\rho_{\frac{1}{2}}\left(\rho_{\frac{5}{2}}+\rho_{\frac{7}{2}}\right)+\rho_{\frac{3}{2}}}+\frac{\rho_{\frac{1}{2}} \rho_{\frac{7}{2}}}{\rho_{\frac{1}{2}} \rho_{\frac{7}{2}}+\rho_{\frac{3}{2}}+\rho_{\frac{5}{2}}}\right) \lambda_{\varnothing}^{t} \\
& a_{\left\{\frac{3}{2}, \frac{7}{2}\right\}}(t)=\frac{\rho_{\frac{3}{2}}}{\rho_{\frac{1}{2}} \rho_{\frac{5}{2}}+\rho_{\frac{3}{2}}}\left(\lambda_{\left\{\frac{3}{2}, \frac{7}{2}\right\}}^{t}-\lambda_{\frac{7}{2}}^{t}\right)-\frac{\rho_{\frac{3}{2}}}{\rho_{\frac{1}{2}}\left(\rho_{\frac{5}{2}}+\rho_{\frac{7}{2}}\right)+\rho_{\frac{3}{2}}}\left(\lambda_{\frac{3}{2}}^{t}-\lambda_{\varnothing}^{t}\right) \\
& a_{\left\{\frac{5}{2}, \frac{7}{2}\right\}}(t)=\lambda_{\left\{\frac{5}{2}, \frac{7}{2}\right\}}^{t}-\lambda_{\frac{7}{2}}^{t}-\frac{\rho_{\frac{5}{2}}}{\rho_{\frac{7}{2}}\left(\rho_{\frac{1}{2}}+\rho_{\frac{3}{2}}\right)+\rho_{\frac{5}{2}}}\left(\lambda_{\frac{5}{2}}^{t}-\lambda_{\varnothing}^{t}\right) \\
& a_{\left\{\frac{1}{2}, \frac{3}{2}, \frac{5}{2}\right\}}(t)=\lambda_{\left\{\frac{1}{2}, \frac{3}{2}, \frac{5}{2}\right\}}^{t}-\lambda_{\left\{\frac{1}{2}, \frac{3}{2}\right\}}^{t}-\frac{\rho_{\frac{5}{2}}}{\rho_{\frac{5}{2}}+\rho_{\frac{3}{2}} \rho_{\frac{7}{2}}}\left(\lambda_{\left\{\frac{1}{2}, \frac{5}{2}\right\}}^{t}-\lambda_{\frac{1}{2}}^{t}\right)-\frac{\rho_{\frac{3}{2}}+\rho_{\frac{5}{2}}}{\rho_{\frac{3}{2}}+\rho_{\frac{5}{2}}+\rho_{\frac{1}{2}} \rho_{\frac{7}{2}}} \lambda_{\left\{\frac{3}{2}, \frac{5}{2}\right\}}^{t} \\
& +\frac{\rho_{\frac{3}{2}}}{\rho_{\frac{1}{2}}\left(\rho_{\frac{5}{2}}+\rho_{\frac{7}{2}}\right)+\rho_{\frac{3}{2}}} \lambda_{\frac{3}{2}}^{t}+\frac{\rho_{\frac{5}{2}}}{\rho_{\frac{7}{2}}\left(\rho_{\frac{1}{2}}+\rho_{\frac{3}{2}}\right)+\rho_{\frac{5}{2}}} \lambda_{\frac{5}{2}}^{t} \\
& -\left(1-\frac{\left(\rho_{\frac{1}{2}}+\rho_{\frac{3}{2}}\right) \rho_{\frac{7}{2}}}{\rho_{\frac{7}{2}}\left(\rho_{\frac{1}{2}}+\rho_{\frac{3}{2}}\right)+\rho_{\frac{5}{2}}}-\frac{\left(\rho_{\frac{5}{2}}+\rho_{\frac{7}{2}}\right) \rho_{\frac{1}{2}}}{\rho_{\frac{1}{2}}\left(\rho_{\frac{5}{2}}+\rho_{\frac{7}{2}}\right)+\rho_{\frac{3}{2}}}+\frac{\rho_{\frac{1}{2}} \rho_{\frac{7}{2}}}{\rho_{\frac{1}{2}} \rho_{\frac{7}{2}}+\rho_{\frac{3}{2}}+\rho_{\frac{5}{2}}}\right) \lambda_{\varnothing}^{t} \\
& a_{\left\{\frac{1}{2}, \frac{3}{2}, \frac{7}{2}\right\}}(t)=\lambda_{\left\{\frac{1}{2}, \frac{3}{2}, \frac{7}{2}\right\}}^{t}-\lambda_{\left\{\frac{1}{2}, \frac{3}{2}\right\}}^{t}-\lambda_{\left\{\frac{1}{2}, \frac{7}{2}\right\}}^{t}-\frac{\rho_{\frac{3}{2}}}{\rho_{\frac{3}{2}}+\rho_{\frac{1}{2}} \rho_{\frac{5}{2}}} \lambda_{\left\{\frac{3}{2}, \frac{7}{2}\right\}}^{t}+\lambda_{\frac{1}{2}}^{t} \\
& +\frac{\rho_{\frac{3}{2}}}{\rho_{\frac{3}{2}}+\rho_{\frac{1}{2}}\left(\rho_{\frac{5}{2}}+\rho_{\frac{7}{2}}\right)} \lambda_{\frac{3}{2}}^{t}+\frac{\rho_{\frac{3}{2}}}{\rho_{\frac{3}{2}}+\rho_{\frac{1}{2}} \rho_{\frac{5}{2}}} \lambda_{\frac{7}{2}}^{t}-\frac{\rho_{\frac{3}{2}}}{\rho_{\frac{3}{2}}+\rho_{\frac{1}{2}}\left(\rho_{\frac{5}{2}}+\rho_{\frac{7}{2}}\right)} \lambda_{\varnothing}^{t} \\
& a_{\left\{\frac{1}{2}, \frac{5}{2}, \frac{7}{2}\right\}}(t)=\lambda_{\left\{\frac{1}{2}, \frac{5}{2}, \frac{7}{2}\right\}}^{t}-\lambda_{\left\{\frac{5}{2}, \frac{7}{2}\right\}}^{t}-\lambda_{\left\{\frac{1}{2}, \frac{7}{2}\right\}}^{t}-\frac{\rho_{\frac{5}{2}}}{\rho_{\frac{5}{2}}+\rho_{\frac{3}{2}} \rho_{\frac{7}{2}}} \lambda_{\left\{\frac{1}{2}, \frac{5}{2}\right\}}^{t}+\lambda_{\frac{7}{2}}^{t} \\
& +\frac{\rho_{\frac{5}{2}}}{\rho_{\frac{5}{2}}+\rho_{\frac{3}{2}} \rho_{\frac{7}{2}}} \lambda_{\frac{1}{2}}^{t}+\frac{\rho_{\frac{5}{2}}}{\rho_{\frac{5}{2}}+\rho_{\frac{7}{2}}\left(\rho_{\frac{1}{2}}+\rho_{\frac{3}{2}}\right)} \lambda_{\frac{5}{2}}^{t}-\frac{\rho_{\frac{5}{2}}}{\rho_{\frac{5}{2}}+\rho_{\frac{7}{2}}\left(\rho_{\frac{1}{2}}+\rho_{\frac{3}{2}}\right)} \lambda_{\varnothing}^{t} \\
& a_{\left\{\frac{3}{2}, \frac{5}{2}, \frac{7}{2}\right\}}(t)=\lambda_{\left\{\frac{3}{2}, \frac{5}{2}, \frac{7}{2}\right\}}^{t}-\lambda_{\left\{\frac{5}{2}, \frac{7}{2}\right\}}^{t}-\frac{\rho_{\frac{3}{2}}}{\rho_{\frac{3}{2}}+\rho_{\frac{1}{2}} \rho_{\frac{5}{2}}}\left(\lambda_{\left\{\frac{3}{2}, \frac{7}{2}\right\}}^{t}-\lambda_{\frac{7}{2}}^{t}\right)-\frac{\rho_{\frac{3}{2}}+\rho_{\frac{5}{2}}}{\rho_{\frac{3}{2}}+\rho_{\frac{5}{2}}+\rho_{\frac{1}{2}} \rho_{\frac{7}{2}}} \lambda_{\left\{\frac{3}{2}, \frac{5}{2}\right\}}^{t} \\
& +\frac{\rho_{\frac{3}{2}}}{\rho_{\frac{1}{2}}\left(\rho_{\frac{5}{2}}+\rho_{\frac{7}{2}}\right)+\rho_{\frac{3}{2}}} \lambda_{\frac{3}{2}}^{t}+\frac{\rho_{\frac{5}{2}}}{\rho_{\frac{7}{2}}\left(\rho_{\frac{1}{2}}+\rho_{\frac{3}{2}}\right)+\rho_{\frac{5}{2}}} \lambda_{\frac{5}{2}}^{t} \\
& -\left(1-\frac{\left(\rho_{\frac{1}{2}}+\rho_{\frac{3}{2}}\right) \rho_{\frac{7}{2}}}{\rho_{\frac{7}{2}}\left(\rho_{\frac{1}{2}}+\rho_{\frac{3}{2}}\right)+\rho_{\frac{5}{2}}}-\frac{\left(\rho_{\frac{5}{2}}+\rho_{\frac{7}{2}}\right) \rho_{\frac{1}{2}}}{\rho_{\frac{1}{2}}\left(\rho_{\frac{5}{2}}+\rho_{\frac{7}{2}}\right)+\rho_{\frac{3}{2}}}+\frac{\rho_{\frac{1}{2}} \rho_{\frac{7}{2}}}{\rho_{\frac{1}{2}} \rho_{\frac{7}{2}}+\rho_{\frac{3}{2}}+\rho_{\frac{5}{2}}}\right) \lambda_{\varnothing}^{t}
\end{aligned}
$$


and

$$
\begin{aligned}
a_{\left\{\frac{1}{2}, \frac{3}{2}, \frac{5}{2}, \frac{7}{2}\right\}}(t)= & \lambda_{\left\{\frac{1}{2}, \frac{3}{2}, \frac{5}{2}, \frac{7}{2}\right\}}^{t}-\lambda_{\left\{\frac{1}{2}, \frac{3}{2}, \frac{5}{2}\right\}}^{t}-\lambda_{\left\{\frac{1}{2}, \frac{3}{2}, \frac{7}{2}\right\}}^{t}-\lambda_{\left\{\frac{1}{2}, \frac{5}{2}, \frac{7}{2}\right\}}^{t}-\lambda_{\left\{\frac{3}{2}, \frac{5}{2}, \frac{7}{2}\right\}}^{t}+\lambda_{\left\{\frac{1}{2}, \frac{3}{2}\right\}}^{t} \\
& +\frac{\rho_{\frac{5}{2}}}{\rho_{\frac{5}{2}}+\rho_{\frac{3}{2}} \rho_{\frac{7}{2}}} \lambda_{\left\{\frac{1}{2}, \frac{5}{2}\right\}}^{t}+\lambda_{\left\{\frac{1}{2}, \frac{7}{2}\right\}}^{t}+\frac{\rho_{\frac{3}{2}}+\rho_{\frac{5}{2}}}{\rho_{\frac{3}{2}}+\rho_{\frac{5}{2}}+\rho_{\frac{1}{2}} \rho_{\frac{7}{2}}} \lambda_{\left\{\frac{3}{2}, \frac{5}{2}\right\}}^{t}+\frac{\rho_{\frac{3}{2}}}{\rho_{\frac{3}{2}}+\rho_{\frac{1}{2}} \rho_{\frac{5}{2}}} \lambda_{\left\{\frac{3}{2}, \frac{7}{2}\right\}}^{t} \\
& +\lambda_{\left\{\frac{5}{2}, \frac{7}{2}\right\}}^{t}-\frac{\rho_{\frac{5}{2}}}{\rho_{\frac{5}{2}}+\rho_{\frac{3}{2}} \rho_{\frac{7}{2}}} \lambda_{\frac{1}{2}}^{t}-\frac{\rho_{\frac{3}{2}}}{\rho_{\frac{1}{2}}\left(\rho_{\frac{5}{2}}+\rho_{\frac{7}{2}}\right)+\rho_{\frac{3}{2}}} \lambda_{\frac{3}{2}}^{t}-\frac{\rho_{\frac{3}{2}}}{\rho_{\frac{7}{2}}\left(\rho_{\frac{1}{2}}+\rho_{\frac{3}{2}}\right)+\rho_{\frac{5}{2}}} \lambda_{\frac{5}{2}}^{t} \\
& -\frac{\rho_{\frac{3}{2}}+\rho_{\frac{1}{2}} \rho_{\frac{5}{2}}}{\rho_{\frac{7}{2}}^{t}} \\
& +\left(1-\frac{\left(\rho_{\frac{5}{2}}+\rho_{\frac{7}{2}}\right) \rho_{\frac{1}{2}}}{\rho_{\frac{1}{2}}\left(\rho_{\frac{5}{2}}+\rho_{\frac{7}{2}}\right)+\rho_{\frac{3}{2}}}-\frac{\left(\rho_{\frac{1}{2}}+\rho_{\frac{3}{2}}\right) \rho_{\frac{7}{2}}}{\rho_{\frac{7}{2}}\left(\rho_{\frac{1}{2}}+\rho_{\frac{3}{2}}\right)+\rho_{\frac{5}{2}}}+\frac{\rho_{\frac{1}{2}} \rho_{\frac{7}{2}}}{\rho_{\frac{1}{2}} \rho_{\frac{7}{2}}+\rho_{\frac{3}{2}}+\rho_{\frac{5}{2}}}\right) \lambda_{\varnothing}^{t},
\end{aligned}
$$

where the $\lambda_{G}$ are given by (42).

\section{References}

1. Aigner, M.: Combinatorial Theory, Springer, Berlin (1979).

2. Baake, M.: Recombination semigroups on measure spaces. Monatsh. Math. 146, 267-278 (2005) and 150, 83-84 (2007) (Addendum); arXiv:math.CA/0506099.

3. Baake, M., Baake, E.: An exactly solved model for mutation, recombination and selection. Can. J. Math. 55 3-41 (2003) and 60, 264-265 (2008) (Erratum); arXiv:math.CA/0210422.

4. Baake, E., Herms, I.: Single-crossover dynamics: finite versus infinite populations. Bull. Math. Biol. 70, 603-624 (2008).

5. Bennett, J. H.: On the theory of random mating. Ann. Human Genetics 18, 311-317 (1954).

6. Christiansen, F. B.: Population Genetics of Multiple Loci. Wiley, Chichester (1999).

7. Cohn, D. L.: Measure Theory. Birkhäuser, Boston (1980).

8. Bürger, R.: The Mathematical Theory of Selection, Recombination and Mutation. Wiley, Chichester (2000).

9. Dawson, K. J.: The decay of linkage disequilibria under random union of gametes: How to calculate Bennett's principal components. Theor. Popul. Biol. 58, 1-20 (2000).

10. Dawson, K. J.: The evolution of a population under recombination: How to linearise the dynamics. Lin. Alg. Appl. 348, 115-137 (2002).

11. Geiringer, H.: On the probability theory of linkage in Mendelian heredity. Ann. Math. Stat. 15, $25-57$ (1944).

12. Hartl, D. L., Clark, A. G.: Principles of Population Genetics. 3rd ed, Sinauer, Sunderland, MA (1997).

13. Jennings, H.S.: The numerical results of diverse systems of breeding, with respect to two pairs of characters, linked or independent, with special relation to the effects of linkage. Genetics 2, 97-154 (1917).

14. Lyubich, Y. I.: Mathematical Structures in Population Genetics. Springer, Berlin (1992).

15. McHale, D., Ringwood, G. A.: Haldane linearisation of baric algebras. J. London Math. Soc. (2) 28, 17-26 (1983).

16. Popa, E.: Some remarks on a nonlinear semigroup acting on positive measures. In: Carja, O., Vrabie, I. I. (Eds.), Applied Analysis and Differential Equations, World Scientific, Singapore, 308-319 (2007).

17. Robbins, R. B.: Some applications of mathematics to breeding problems III. Genetics 3, 375-389 (1918).

18. von Wangenheim, U.: Diskrete Rekombinationsdynamik. Diplomarbeit, Universität Greifswald (2007). 